July 29, 2018

\title{
INFINITE HORIZON STOCHASTIC OPTIMAL CONTROL PROBLEMS WITH RUNNING MAXIMUM COST
}

\author{
AXEL KRÖNER, ATHENA PICARELLI, AND HASNAA ZIDANI
}

\begin{abstract}
An infinite horizon stochastic optimal control problem with running maximum cost is considered. The value function is characterized as the viscosity solution of a second-order Hamilton-Jacobi-Bellman (HJB) equation with mixed boundary condition. A general numerical scheme is proposed and convergence is established under the assumptions of consistency, monotonicity and stability of the scheme. These properties are verified for a specific semi-Lagrangian scheme.
\end{abstract}

\section{INTRODUCTION}

In this paper we consider infinite horizon stochastic optimal control problems with cost in a maximum form of the following type:

$$
\left\{\begin{aligned}
\inf _{u \in \mathcal{U}} \mathbb{E}\left[\max _{s \in[0, \infty)} e^{-\lambda s} g\left(X_{x}^{u}(s)\right)\right], \quad \text { subject to } \\
\mathrm{d} X_{x}^{u}(s)=b\left(X_{x}^{u}(s), u(s)\right) \mathrm{d} s+\sigma\left(X_{x}^{u}(s), u(s)\right) \mathrm{d} B(s), \quad s \in[0, \infty), \\
X_{x}^{u}(0)=x \in \mathbb{R}^{d}
\end{aligned}\right.
$$

with $p$-dimensional Brownian motion $B(\cdot)$. The control $u$ belongs to a set $\mathcal{U}$ of progressively measurable processes with values in a given compact set $U \subset \mathbb{R}^{m}$. The functions $g: \mathbb{R}^{d} \rightarrow \mathbb{R}, b: \mathbb{R}^{d} \times U \rightarrow \mathbb{R}^{d}$ and $\sigma: \mathbb{R}^{d} \times U \rightarrow \mathbb{R}^{d \times p}$, and the discount factor $\lambda>0$ are supposed to be known. Control problems of this type can be used for the characterization of viable and invariant sets and arise in the study of some path-dependent options in finance (lookback, Russian options). The study of this problem is also motivated by some engineering applications, see for instance $[29,3]$.

In the deteministic case, problems with supremum costs and finite time horizon have been extensively studied in the literature, we refer for instance to $[8,9,2,10]$ where the value function is characterized as unique solution of a Hamilton-Jacobi-Bellman (HJB) equation with an obstacle. The value function is also analysed within the viability framework in [34, 35], and linearization techniques for such problems are presented in [23].

The case of deterministic control problems with supremum costs and infinite horizon has been also considered in $[8,20,21]$. Note that in this case it is shown that the value function satisfies a stationary HJB equation with an obstacle. However, unlike the finite horizon case, the HJB equation

The first author acknowledges the hospitality and support of the COMMANDS team at ENSTA ParisTech (Palaiseau, France) where, during a research visit, this project started. 
may have several viscosity solutions. The only characterization of the value function is obtained by the optimality principles derived from the dynamic programming principle (DPP). In [1, 21], a control problem without discount factor is considered and the value function of such a problem is shown to be the limit of value functions associated to some control problems with maximum running cost in finite horizon.

In the stochastic setting with finite time horizon, the characterization of the value function is studied in $[4,5,7,8,11]$. We refer also to [26] where some special cases of control problems with running costs are solved by using the DPP. We point out that the stochastic framework presents specific difficulties coming from the non commutativity between the expectation and the maximum operator. In $[4,5,7,8]$ the characterization of the value function is obtained by considering an $L^{p}$ approximation technique where the maximum cost is approximated by a sequence of $L^{p}$ costs using the fact that for any $a, b \geq 0$

$$
\max (a, b) \simeq\left(a^{p}+b^{p}\right)^{\frac{1}{p}} \quad(\text { for } p \rightarrow \infty) .
$$

Note that this technique can be used only when the cost function $g$ is positive. In [11], the DPP and the HJB equation are derived directly for the maximum running cost problem without using the $L^{p}$ approximation. In addition, a numerical scheme based on a Semi-Lagrangian (SL) approach is analysed for the computation of the value function.

In the present work, we consider the case of stochastic control problem with supremum running cost in infinite horizon. The cost function involves also a discount factor $\lambda$ that is positive. We are interested in the characterization of the value function and its numerical approximation. By using the same viscosity arguments as in [11], we show that the value function satisfies a HJB equation with a boundary condition involving an oblique derivative. Unlike the finite time horizon case (see $[4,7,11]$ ), this HJB equation fails to be a good characterization of the value function as it may admit several trivial solutions.

To garantee the uniqueness, we complete the HJB system by a Dirichlet boundary condition. While the oblique derivative boundary condition is understood in the viscosity sense [28], the Dirichlet condition is considered pointwise. A similar idea has been also used in [25], however in that paper, the uniqueness result is proved by using some arguments of nonsmooth analysis that are valid only under some strong stability assumptions on the differential process. Here, we use only PDE arguments and prove the uniqueness result without assuming additional assumptions on the state.

The second part of the paper is devoted to the numerical approximation. Thereby we follow some ideas developed in [11] for the corresponding finite horizon problem. The extension is not straightforward since we are now dealing with a stationary equation with mixed boundary conditions. 
First, by using the framework of Barles-Souganidis [6], we obtain a convergence result for a general class of numerical schemes satisfying some adequate monotonicity, stability and consistency properties. Then, we introduce a SL scheme and prove its convergence. Recall that SL schemes have been introduced in [16] for first order Hamilton-Jacobi equations and then extended to the second order case in $[15,18,19,32,33]$. For time-dependent equations with an oblique boundary condition (that appears in the case of finite horizon control problems), the SL method has been investigated in [11].

For the stationary equations as considered in the present work, the SL schemes are formulated as fixed-point problems. Let us point out that the presence of mixed boundary conditions arises some new difficulties in the analysis of this fixed point problem. To deal with these difficulties, the numerical scheme we propose couples the classical SL scheme with additional projection steps on the boundary taking into account the overstepping of the domain which is typical in such a wide stencil scheme. We prove that our scheme is stable, consistent and monotone. We analyse also the fixed-point operator in an adequate space where the fixed-point algorithm converges.

The paper is organized as follows: Section 2 introduces the problem and the main assumptions. Sections 3 and 4 are devoted to the characterization of the value function by the appropriate HJB equation: the DPP is established, the HJB equation is derived and uniqueness proven by a strong comparison principle. In Section 5 the numerical approximation is discussed and a general convergence result is provided. The SL scheme is presented in Section 5.2 and its convergence properties are investigated. A numerical test in two dimensions is presented in Section 6.

\section{Formulation of the Problem}

Let $(\Omega, \mathcal{F}, \mathbb{P})$ be a probability space, $\left\{\mathcal{F}_{t}, t \geq 0\right\}$ a filtration on $\mathcal{F}$ and $B(\cdot)$ a $\left\{\mathcal{F}_{t}\right\}_{t>0}$-Brownian motion in $\mathbb{R}^{p}, p \geq 1$. Let $\mathcal{U}$ be a set of progressively measurable processes with values in a compact set $U \subset \mathbb{R}^{m}$ (with $m \geq 1$ ). For every control input $u \in \mathcal{U}$, and every $x \in \mathbb{R}^{d}$, we consider the stochastic differential equation:

$$
\left\{\begin{aligned}
\mathrm{d} X_{x}^{u}(s) & =b\left(X_{x}^{u}(s), u(s)\right) \mathrm{d} s+\sigma\left(X_{x}^{u}(s), u(s)\right) \mathrm{d} B(s), \quad s \in[0, \infty), \\
X_{x}^{u}(0) & =x
\end{aligned}\right.
$$

Throughout the paper we make the following assumptions on the coefficients in $(2.1)$ :

(H1) $b: \mathbb{R}^{d} \times U \rightarrow \mathbb{R}^{d}$ and $\sigma: \mathbb{R}^{d} \times U \rightarrow \mathbb{R}^{d \times p}$ are continuous functions. There exists $C_{0} \geq 0$ such that for any $x, y \in \mathbb{R}^{d}$ and $u \in U$, we have:

$$
|b(x, u)-b(y, u)|+|\sigma(x, u)-\sigma(y, u)| \leq C_{0}|x-y| .
$$

Proposition 2.1. Let assumption (H1) hold. Then, for any $x \in \mathbb{R}^{d}$ and $u \in \mathcal{U}$ there exists a unique strong solution $X_{x}^{u}(\cdot)$ of $(2.1)$. Moreover, there 
exists $C \geq 0$ such that

$$
\mathbb{E}\left[\max _{\theta \in[0, T]}\left|X_{x}^{u}(\theta)-X_{x^{\prime}}^{u}(\theta)\right|\right] \leq C e^{C T}\left|x-x^{\prime}\right|
$$

for any $u \in \mathcal{U}, T>0$ and $x, x^{\prime} \in \mathbb{R}^{d}$.

The proof of the above result can be found in [37, p. 42, Thm. 6.3] and [36, p.14]. Now, consider a cost function $g$ satisfying the following condition:

(H2) $g: \mathbb{R}^{d} \rightarrow \mathbb{R}$ is Lipschitz continuous and bounded, i.e., there exist $M_{g}, L_{g} \geq 0$ such that

$$
|g(x)-g(y)| \leq L_{g}|x-y|, \quad|g(x)| \leq M_{g}, \quad \forall x, y \in \mathbb{R}^{d} .
$$

For given discount factor $\lambda>0$ and initial position $x \in \mathbb{R}^{d}$ the infinite horizon optimal control problem reads as

$$
\inf _{u \in \mathcal{U}} J(x, u):=\mathbb{E}\left[\max _{s \in[0, \infty)} e^{-\lambda s} g\left(X_{x}^{u}(s)\right)\right] .
$$

We will denote by $v: \mathbb{R}^{d} \rightarrow \mathbb{R}$ the associated value function, i.e.

$$
v(x):=\inf _{u \in \mathcal{U}} J(x, u)
$$

\section{Dynamic Programming Principle}

In the next sections, we aim to find a characterization of the value function $v$ as solution of a PDE. The presence of the maximum operator inside the expectation makes the cost $J$ in (2.3) non-Markovian and it is wellknown that this prevents to establish a DPP, which is the first fundamental result towards the HJB characterisation. A classical strategy to overcome this difficulty consists in adding an auxiliary variable $y$ that, roughly speaking, gets rid of the non-Markovian component of the cost. Let us define the auxiliary value function $\vartheta: \mathbb{R}^{d} \times \mathbb{R} \rightarrow \mathbb{R}$

$$
\vartheta(x, y):=\inf _{u \in \mathcal{U}} \mathbb{E}\left[\max _{s \in[0, \infty)} e^{-\lambda s} g\left(X_{x}^{u}(s)\right) \vee y\right],
$$

which satisfies, for any $x \in \mathbb{R}^{d}$,

$$
\vartheta(x, g(x))=v(x)
$$

where $v$ is defined as in (2.4). Consequently, if the value function $\vartheta$ of the auxiliary problem is known, one can immediately recover the original value function $v$. Therefore, we consider only $\vartheta$ in the sequel of the paper.

We start by proving a continuity result for the auxiliary value function.

Proposition 3.1. Let assumptions (H1)-(H2) be satisfied. Then the value function $\vartheta$ is uniformly continuous in $\mathbb{R}^{d} \times \mathbb{R}$. Moreover,

$$
\vartheta\left(x, M_{g}\right)=M_{g} \quad \text { and } \quad|\vartheta(x, y)| \leq M_{g}
$$

for any $(x, y) \in \mathbb{R}^{d} \times \mathbb{R}$ with $|y| \leq M_{g}$. 
Proof. The last statement follows directly by the definition of $\vartheta$ and the bound on $g$ given by assumption (H2).

By the very definition of $\vartheta$ we have that for any $(x, y),(\xi, \eta) \in \mathbb{R}^{d} \times \mathbb{R}$

$$
\begin{aligned}
& |\vartheta(x, y)-\vartheta(\xi, \eta)| \\
& \leq \sup _{u \in \mathcal{U}} \mathbb{E}\left[\left|\max _{s \in[0, \infty)} e^{-\lambda s} g\left(X_{x}^{u}(s)\right) \vee y-\max _{s \in[0, \infty)} e^{-\lambda s} g\left(X_{\xi}^{u}(s)\right) \vee \eta\right|\right] \\
& \leq \sup _{u \in \mathcal{U}} \mathbb{E}\left[\max _{s \in[0, \infty)}\left|e^{-\lambda s} g\left(X_{x}^{u}(s)\right)-e^{-\lambda s} g\left(X_{\xi}^{u}(s)\right)\right| \vee|y-\eta|\right] \\
& \leq \sup _{u \in \mathcal{U}} \mathbb{E}\left[\max _{s \in[0, \infty)}\left|e^{-\lambda s} g\left(X_{x}^{u}(s)\right)-e^{-\lambda s} g\left(X_{\xi}^{u}(s)\right)\right|\right]+|y-\eta|
\end{aligned}
$$

For any $T>0$ we have that (recalling that $g$ is bounded)

$$
\begin{aligned}
& \mathbb{E}\left[\max _{s \in[0, \infty)} e^{-\lambda s}\left|g\left(X_{x}^{u}(s)\right)-g\left(X_{\xi}^{u}(s)\right)\right|\right] \\
& \leq \mathbb{E}\left[\max _{s \in[0, T]} e^{-\lambda s}\left|g\left(X_{x}^{u}(s)\right)-g\left(X_{\xi}^{u}(s)\right)\right|+\max _{s \in[T, \infty)} e^{-\lambda s}\left|g\left(X_{x}^{u}(s)\right)-g\left(X_{\xi}^{u}(s)\right)\right|\right] \\
& \leq \mathbb{E}\left[\max _{s \in[0, T]} e^{-\lambda s}\left|g\left(X_{x}^{u}(s)\right)-g\left(X_{\xi}^{u}(s)\right)\right|\right]+\mathbb{E}\left[\max _{s \in[T, \infty)} 2 M_{g} e^{-\lambda s}\right] \\
& =\mathbb{E}\left[\max _{s \in[0, T]} e^{-\lambda s}\left|g\left(X_{x}^{u}(s)\right)-g\left(X_{\xi}^{u}(s)\right)\right|\right]+2 M_{g} e^{-\lambda T} .
\end{aligned}
$$

For the first term we have the classical estimates (see Theorem 2.1)

$$
\mathbb{E}\left[\max _{s \in[0, T]} e^{-\lambda s}\left|g\left(X_{x}^{u}(s)\right)-g\left(X_{\xi}^{u}(s)\right)\right|\right] \leq L_{g} C e^{C T}|x-\xi| .
$$

So putting everything together we get that for every $T$

$$
|\vartheta(x, y)-\vartheta(\xi, \eta)| \leq L_{g} C e^{C T}|x-\xi|+2 M_{g} e^{-\lambda T}+|y-\eta| .
$$

Now, for any $\varepsilon>0$ we can fix $T \equiv T(\varepsilon)$ such that $2 M_{g} e^{-\lambda T}<\varepsilon / 3$. It follows that we can choose a $\delta=\delta(\varepsilon, T(\varepsilon))$ such that if $|(x, y)-(\xi, \eta)| \leq \delta$ then

$$
|\vartheta(x, y)-\vartheta(\xi, \eta)|<\varepsilon
$$

Remark 3.2. In many cases, under assumptions (H1)-(H2), the value function is expected to be Hölder continuous. This holds true in the case of finite horizon problems for both integral and maximum running costs (see [37, Proposition 3.1] and [11, Proposition 2.2], respectively). In infinite time horizon, such a property can be recovered only for a discount factor sufficiently big ensuring a dissipative property of the process $e^{-\lambda t} X_{x}^{u}(t)$ (see for instance [31, Theorem 3.4] for the case of integral running cost). However, the proof of Proposition 3.1 shows that $\vartheta$ is Lipschitz continuous with respect to the auxiliary variable $y$. This will turn out to be a fundamental property to 
be preserved when defining a numerical scheme in order to get convergence (see assumption (H3.a) in Section 5).

Thanks to the presence of the auxiliary variable $y$ we are able to state the DPP for $\vartheta$. Let us define for $t \geq 0$

$$
Y_{x, y}^{u}(t):=\max _{s \in[0, t]} e^{-\lambda s} g\left(X_{x}^{u}(s)\right) \vee y .
$$

Following the arguments in [14] and thanks to the uniform continuity of $\vartheta$ one has:

Theorem 3.3 (DPP). Let assumptions (H1)-(H2) be satisfied. For $(x, y) \in$ $\mathbb{R}^{d} \times \mathbb{R}$ and any finite stopping time $\theta \geq 0$ there holds

$$
\vartheta(x, y)=\inf _{u \in \mathcal{U}} \mathbb{E}\left[e^{-\lambda \theta} \vartheta\left(X_{x}^{u}(\theta), e^{\lambda \theta} Y_{x, y}^{u}(\theta)\right)\right] .
$$

Proof. The proof of the DPP is based on the fact that for every $u \in \mathcal{U}$, we have:

$$
\begin{aligned}
\max _{s \in[0, \infty)} e^{-\lambda s} g\left(X_{x}^{u}(s)\right) \vee y & =\max _{s \in[\theta, \infty)} e^{-\lambda s} g\left(X_{x}^{u}(s)\right) \bigvee \underbrace{\max _{s \in[0, \theta]} e^{-\lambda s} g\left(X_{x}^{u}(s)\right) \vee y}_{=: Y_{x, y}(\theta)} \\
& =e^{-\lambda \theta} \max _{s \in[0, \infty)} e^{-\lambda s} g\left(X_{X_{x}^{u}(\theta)}^{u}(s)\right) \bigvee Y_{x, y}(\theta) \\
& =e^{-\lambda \theta}\left(\max _{s \in[0, \infty)} e^{-\lambda s} g\left(X_{X_{x}^{u}(\theta)}^{u}(s)\right) \bigvee e^{\lambda \theta} Y_{x, y}(\theta)\right)
\end{aligned}
$$

By the tower property of the expectation, we conclude the proof. We refer to [14] and [36] for more detailed arguments.

\section{The Hamilton-Jacobi-Bellman equation}

The value function $\vartheta$ can be characterized in terms of a solution of a second-order HJB equation.

Theorem 4.1 (Second-order HJB equation). Under assumptions (H1)(H2), the value function $\vartheta$ is a continuous viscosity solution of

$$
\begin{array}{rlrl}
\lambda \vartheta+H\left(x, y, D_{x} \vartheta, \partial_{y} \vartheta, D_{x}^{2} \vartheta\right) & =0 & \text { for } x & \in \mathbb{R}^{d}, y>g(x), \\
-\partial_{y} \vartheta & =0 & \text { for } x \in \mathbb{R}^{d}, y=g(x)
\end{array}
$$

with $\lambda>0$ and Hamiltonian $H: \mathbb{R}^{d} \times \mathbb{R} \times \mathbb{R}^{d} \times \mathbb{R} \times \mathbb{R}^{d \times d} \rightarrow \mathbb{R}$ defined by

$$
H(x, y, p, q, P)=\sup _{u \in U}\left(-b(x, u) \cdot p-\frac{1}{2} \operatorname{Tr}\left[\left(\sigma \sigma^{T}\right)(x, u) P\right]\right)-\lambda y q .
$$

We recall the notion of viscosity solution for second-order HJB equation.

Definition 4.2. [17, Definition 7.4] Let $\mathcal{O}$ be a locally compact set in $\mathbb{R}^{d+1}$. A USC function $\underline{\vartheta}$ (resp. LSC function $\bar{\vartheta}$ ) on $\overline{\mathcal{O}}$ is a viscosity sub-solution (resp. super-solution) of (4.1), if for every function $\varphi \in C^{2}(\overline{\mathcal{O}})$ at every 
maximum (resp. minimum) point $x$ of $\underline{\vartheta}-\varphi($ resp. $\bar{\vartheta}-\varphi)$ the following inequalities hold

$$
\left\{\begin{aligned}
\lambda \varphi+H\left(x, y, D_{x} \varphi, \partial_{y} \varphi, D_{x}^{2} \varphi\right) \leq 0 & \text { in } \mathcal{O} \\
\min \left(\lambda \varphi+H\left(x, y, D_{x} \varphi, \partial_{y} \varphi, D_{x}^{2} \varphi\right),-\partial_{y} \varphi\right) & \leq 0 \quad \text { on } \partial \mathcal{O}
\end{aligned}\right.
$$

(resp.

$$
\left\{\begin{aligned}
\lambda \varphi+H\left(x, y, D_{x} \varphi, \partial_{y} \varphi, D_{x}^{2} \varphi\right) & \geq 0 \quad \text { in } \mathcal{O}, \\
\max \left(\lambda \varphi+H\left(x, y, D_{x} \varphi, \partial_{y} \varphi, D_{x}^{2} \varphi\right),-\partial_{y} \varphi\right) \geq 0 & \text { on } \partial \mathcal{O} .)
\end{aligned}\right.
$$

A continuous function $\vartheta$ is called a viscosity solution of (4.1) if it is both a sub- and super-solution.

Proof of Theorem 4.1. Let

$$
\overline{\mathcal{O}}:=\left\{(x, y) \in \mathbb{R}^{d+1}: y \geq g(x)\right\} .
$$

We first check that $\vartheta$ is a viscosity sub-solution. Let $\varphi \in C^{2}(\overline{\mathcal{O}})$ such that $\vartheta-\varphi$ attains a maximum at point $(\bar{x}, \bar{y}) \in \overline{\mathcal{O}}$. Without loss of generality we can assume that $(\bar{x}, \bar{y})$ is a strict local maximum point (let us say in a ball of radius $r>0$ centered in $(\bar{x}, \bar{y}))$ and $\vartheta(\bar{x}, \bar{y})=\varphi(\bar{x}, \bar{y})$. Thanks to Theorem 3.3, for any $u \in \mathcal{U}$ and for any sufficiently small stopping time $\theta=\theta^{u}$, we have:

$$
\begin{aligned}
\varphi(\bar{x}, \bar{y}) & =\vartheta(\bar{x}, \bar{y}) \leq \mathbb{E}\left[e^{-\lambda \theta} \vartheta\left(X_{\bar{x}}^{u}(\theta), e^{\lambda \theta} Y_{\bar{x}, \bar{y}}^{u}(\theta)\right)\right] \\
& \leq \mathbb{E}\left[e^{-\lambda \theta} \varphi\left(X_{\bar{x}}^{u}(\theta), e^{\lambda \theta} Y_{\bar{x}, \bar{y}}^{u}(\theta)\right)\right] .
\end{aligned}
$$

Two cases will be considered depending on if the point $(\bar{x}, \bar{y})$ belongs to the boundary of $\overline{\mathcal{O}}$ or not.

- Case 1: $g(\bar{x})<\bar{y}$. Consider a constant control $u(s) \equiv u \in U$. From the continuity of $g$ and the a.s. continuity of the sample paths it follows that for a.e. $\omega \in \Omega$ there exists $\bar{s}(\omega)>0$ such that $g\left(X_{\bar{x}}^{u}(s)\right) e^{-\lambda s}<\bar{y}$ if $s \in[0, \bar{s}(\omega))$. Given $h>0$, let $\bar{\theta}$ be the following stopping time:

$$
\begin{aligned}
\bar{\theta}:= & \inf \left\{s>0:\left(X_{\bar{x}}^{u}(s), e^{\lambda s} Y_{\bar{x}, \bar{y}}^{u}(s)\right) \notin B((\bar{x}, \bar{y}), r)\right\} \wedge h \\
& \wedge \inf \left\{s>0: e^{-\lambda s} g\left(X_{\bar{x}}^{u}(s)\right) \geq \bar{y}\right\}
\end{aligned}
$$

(where $B((\bar{x}, \bar{y}), r)$ denotes the ball of radius $r>0$ centered at $(\bar{x}, \bar{y})$ and $a \wedge b=\min (a, b))$. One can easily observe that a.s. $\bar{\theta}>0$ and $Y_{\bar{x}, \bar{y}}^{u}(\bar{\theta})=\bar{y}$, then by $(4.3)$

$$
\varphi(\bar{x}, \bar{y}) \leq \mathbb{E}\left[e^{-\lambda \bar{\theta}} \varphi\left(X_{\bar{x}}^{u}(\bar{\theta}), e^{\lambda \bar{\theta}} \bar{y}\right)\right] \quad \forall u \in U
$$


By applying Ito's formula [37, Theorem 5.5], and thanks to the smoothness of $\varphi$, we get:

$$
\begin{aligned}
0 \geq & \mathbb{E}\left[\int_{0}^{\bar{\theta}}-\mathrm{d}\left(e^{-\lambda s} \varphi\left(X_{\bar{x}}^{u}(s), e^{\lambda s} \bar{y}\right)\right)\right] \\
= & \mathbb{E}\left[\int _ { 0 } ^ { \overline { \theta } } \left\{\lambda \varphi\left(X_{\bar{x}}^{u}(s), e^{\lambda s} \bar{y}\right)-b\left(X_{\bar{x}}^{u}(s), u\right) D_{x} \varphi\left(X_{\bar{x}}^{u}(s), e^{\lambda s} \bar{y}\right)\right.\right. \\
& \left.\left.-\frac{1}{2} \operatorname{Tr}\left[\sigma \sigma^{T}\left(X_{\bar{x}}^{u}(s), u\right) D_{x}^{2} \varphi\left(X_{\bar{x}}^{u}(s), e^{\lambda s} \bar{y}\right)\right]-\lambda \bar{y} e^{\lambda s} \partial_{y} \varphi\left(X_{\bar{x}}^{u}(s), e^{\lambda s} \bar{y}\right)\right\} e^{-\lambda s} d s\right] .
\end{aligned}
$$

Observing that the stopping times

$\inf \left\{s>0:\left(X_{\bar{x}}^{u}(s), e^{\lambda s} Y_{\bar{x}, \bar{y}}^{u}(s)\right) \notin B_{r}(\bar{x}, \bar{y})\right\}$ and $\inf \left\{s>0: e^{-\lambda s} g\left(X_{\bar{x}}^{u}(s)\right) \geq \bar{y}\right\}$

are a.s. strictly greater than 0 , for a.e. fixed $\omega$ one obtains $\bar{\theta}=h$ for a sufficiently small $h$ in (4.4). Dividing by $h>0$, one gets

$$
\begin{aligned}
\mathbb{E} & {\left[\frac { 1 } { h } \int _ { 0 } ^ { \overline { \theta } } \left\{\lambda \varphi\left(X_{\bar{x}}^{u}(s), e^{\lambda s} \bar{y}\right)-b\left(X_{\bar{x}}^{u}(s), u\right) D_{x} \varphi\left(X_{\bar{x}}^{u}(s), e^{\lambda s} \bar{y}\right)\right.\right.} \\
& \left.\left.-\frac{1}{2} \operatorname{Tr}\left[\sigma \sigma^{T}\left(X_{\bar{x}}^{u}(s), u\right) D_{x}^{2} \varphi\left(X_{\bar{x}}^{u}(s), e^{\lambda s} \bar{y}\right)\right]-\lambda \bar{y} e^{\lambda s} \partial_{y} \varphi\left(X_{\bar{x}}^{u}(s), e^{\lambda s} \bar{y}\right)\right\} e^{-\lambda s} d s\right] \leq 0 .
\end{aligned}
$$

By the dominate convergence theorem, taking the limit for $h$ going to 0 inside the expectation and applying the mean value theorem, it follows

$$
\lambda \varphi(\bar{x}, \bar{y})-b(\bar{x}, u) D_{x} \varphi(\bar{x}, \bar{y})-\frac{1}{2} \operatorname{Tr}\left[\sigma \sigma^{T}(\bar{x}, u) D_{x}^{2} \varphi(\bar{x}, \bar{y})\right]-\lambda \bar{y} \partial_{y} \varphi(\bar{x}, \bar{y}) \leq 0 .
$$

Finally, thanks to the arbitrariness of $u \in U$ :

$$
\lambda \varphi(\bar{x}, \bar{y})+\sup _{u \in U}\left(-b(\bar{x}, u) D_{x} \varphi(\bar{x}, \bar{y})-\frac{1}{2} \operatorname{Tr}\left[\sigma \sigma^{T}(\bar{x}, u) D_{x}^{2} \varphi(\bar{x}, \bar{y})\right]\right)-\lambda \bar{y} \partial_{y} \varphi(\bar{x}, \bar{y}) \leq 0 .
$$

- Case 2: $g(\bar{x})=\bar{y}$. Let us assume that $-\partial_{y} \varphi(\bar{x}, \bar{y})>0$, otherwise the conclusion is straightforward.

As in the previous case, we consider a constant control $u(s) \equiv u \in U$. Thanks to the continuity of the sample paths and the smoothness of $\varphi$, for a.e. $\omega$ there is a time $\bar{s}(\omega)>0$ and $\eta>0$ such that:

$$
\varphi\left(X_{\bar{x}}^{u}(s), e^{\lambda s} y\right) \leq \varphi\left(X_{\bar{x}}^{u}(s), e^{\lambda s} \bar{y}\right) \quad \forall s \in[0, \bar{s}], y \in[\bar{y}, \bar{y}+\eta) .
$$

Let $\bar{\theta}$ be the stopping time given by:

$$
\begin{aligned}
\bar{\theta}:= & \inf \left\{s>0:\left(X_{\bar{x}}^{u}(s), e^{\lambda s} Y_{\bar{x}, \bar{y}}^{u}(s)\right) \notin B((\bar{x}, \bar{y}), r)\right\} \wedge \inf \left\{s>0: \partial_{y} \varphi\left(X_{\bar{x}}^{u}(s), \bar{y}\right) \geq 0\right\} \\
& \wedge \inf \left\{s>0: e^{-\lambda s} g\left(X_{\bar{x}}^{u}(s)\right) \notin[\bar{y}, \bar{y}+\eta)\right\} \wedge h .
\end{aligned}
$$

By (4.3) we have $\varphi(\bar{x}, \bar{y}) \leq \mathbb{E}\left[e^{-\lambda \bar{\theta}} \varphi\left(X_{\bar{x}}^{u}(\bar{\theta}), e^{\lambda \bar{\theta}} \bar{y}\right)\right]$, which implies (as in Case 1):

$$
\lambda \varphi(\bar{x}, \bar{y})+\sup _{u \in U}\left\{-b(\bar{x}, u) D_{x} \varphi(\bar{x}, \bar{y})-\frac{1}{2} \operatorname{Tr}\left[\sigma \sigma^{T}(\bar{x}, u) D_{x}^{2} \varphi(\bar{x}, \bar{y})\right]\right\}-\lambda \bar{y} \partial_{y} \varphi(\bar{x}, \bar{y}) \leq 0 .
$$


In conclusion at $(\bar{x}, \bar{y}) \in \partial \mathcal{O}$ we have

$$
\min \left(\lambda \varphi+H\left(\bar{x}, \bar{y}, D_{x} \varphi, \partial_{y} \varphi, D_{x}^{2} \varphi\right),-\partial_{y} \varphi\right) \leq 0,
$$

and $\vartheta$ is a viscosity sub-solution of equation (4.1).

It remains to prove that $\vartheta$ is a viscosity super-solution of (4.1). Let $\varphi \in C^{2}(\overline{\mathcal{O}})$ be such that $\vartheta-\varphi$ attains a minimum at point $(\bar{x}, \bar{y}) \in \overline{\mathcal{O}}$. Without loss of generality we can always assume that $(\bar{x}, \bar{y})$ is a strict local minimum point in a ball $B((\bar{x}, \bar{y}), r)$ and $\vartheta(\bar{x}, \bar{y})=\varphi(\bar{x}, \bar{y})$. We consider again the two cases:

- Case 1: $g(\bar{x})<\bar{y}$. We assume by contradiction that

$$
\lambda \varphi+H\left(\bar{x}, \bar{y}, D_{x} \varphi, \partial_{y} \varphi, D_{x}^{2} \varphi\right)<0 .
$$

By using continuity arguments we can also state that

$$
\lambda \varphi+H\left(\cdot, \cdot, D_{x} \varphi, \partial_{y} \varphi, D_{x}^{2} \varphi\right) \leq 0
$$

in a neighborhood $B\left((\bar{x}, \bar{y}), r_{1}\right)$ of $(\bar{x}, \bar{y})$ for some $r_{1}>0$. Moreover, thanks to the continuity of $g$, if $\bar{y}-g(\bar{x})=: \rho>0$ we can find $r_{2}(\rho)>0$ and $T(\rho)>0$ such that

$$
\max _{\substack{t \in[0, T], x \in B\left(\bar{x}, r_{2}\right)}} e^{-\lambda t} g(x)-g(\bar{x}) \leq \frac{\rho}{2}
$$

and we have

$$
\max _{\substack{t \in[0, T], x \in B\left(\bar{x}, r_{2}\right)}} e^{-\lambda t} g(x) \vee \bar{y}=\bar{y} .
$$

For any $u \in \mathcal{U}$ we define the stopping time $\theta^{u}$ as the first exit time of the process $\left(X_{\bar{x}}^{u}(s), e^{\lambda s} Y_{\bar{x}, \bar{y}}^{u}(s)\right)$ from the ball $B((\bar{x}, \bar{y}), R)$ for $R:=\min \left(r, r_{1}, r_{2}\right)>0$, i.e.

$$
\theta^{u}:=\inf \left\{s>0:\left(X_{\bar{x}}^{u}(s), e^{\lambda s} Y_{\bar{x}, \bar{y}}^{u}(s)\right) \notin B((\bar{x}, \bar{y}), R)\right\} \wedge T .
$$

Applying Ito's formula and taking the expectation we get

$$
\begin{aligned}
& \varphi(\bar{x}, \bar{y})-\mathbb{E}\left[e^{-\lambda \theta^{u}} \varphi\left(X_{\bar{x}}^{u}\left(\theta^{u}\right), e^{\lambda \theta^{u}} Y_{\bar{x}, \bar{y}}^{u}\left(\theta^{u}\right)\right)\right] \\
& =\varphi(\bar{x}, \bar{y})-\mathbb{E}\left[e^{-\lambda \theta^{u}} \varphi\left(X_{\bar{x}}^{u}\left(\theta^{u}\right), e^{\lambda \theta^{u}} \bar{y}\right)\right] \\
& =\mathbb{E}\left[\int _ { 0 } ^ { \theta ^ { u } } \left\{\lambda \varphi\left(X_{\bar{x}}^{u}(s), e^{\lambda s} \bar{y}\right)-b\left(X_{\bar{x}}^{u}(s), u\right) D_{x} \varphi\left(X_{\bar{x}}^{u}(s), e^{\lambda s} \bar{y}\right)\right.\right. \\
& \left.\left.\quad-\frac{1}{2} \operatorname{Tr}\left[\sigma \sigma^{T}\left(X_{\bar{x}}^{u}(s), u\right) D_{x}^{2} \varphi\left(X_{\bar{x}}^{u}(s), e^{\lambda s} \bar{y}\right)\right]-\lambda \bar{y} e^{\lambda s} \partial_{y} \varphi\left(X_{\bar{x}}^{u}(s), e^{\lambda s} \bar{y}\right)\right\} e^{-\lambda s} d s\right] \leq 0,
\end{aligned}
$$

that leads to

$$
\vartheta(\bar{x}, \bar{y})=\varphi(\bar{x}, \bar{y}) \leq \mathbb{E}\left[e^{-\lambda \theta^{u}} \varphi\left(X_{\bar{x}}^{u}\left(\theta^{u}\right), e^{\lambda \theta^{u}} Y_{\bar{x}, \bar{y}}^{u}\left(\theta^{u}\right)\right)\right] .
$$


The continuity of the sample paths implies that

$$
\left\{\begin{array}{l}
\text { either } \theta^{u}=T \text { or } \\
\left(X_{\bar{x}}^{u}\left(\theta^{u}\right), e^{\lambda \theta^{u}} Y_{\bar{x}, \bar{y}}^{u}\left(\theta^{u}\right)\right) \in \partial B((\bar{x}, \bar{y}), R) \text { a.s. }
\end{array}\right.
$$

Since $(\bar{x}, \bar{y})$ is a strict minimum point we have

$\min \left\{(\vartheta-\varphi):(x, y) \in \partial B((\bar{x}, \bar{y}), R) \bigcup\left(B((\bar{x}, \bar{y}), R) \cap y=e^{\lambda T} \bar{y}\right)\right\}=: \eta>0$, and hence

$$
\vartheta(\bar{x}, \bar{y}) \leq \varphi\left(X_{\bar{x}}^{u}\left(\theta^{u}\right), e^{\lambda \theta^{u}} Y_{\bar{x}, \bar{y}}^{u}\left(\theta^{u}\right)\right) \leq \vartheta\left(X_{\bar{x}}^{u}\left(\theta^{u}\right), e^{\lambda \theta^{u}} Y_{\bar{x}, \bar{y}}^{u}\left(\theta^{u}\right)\right)-\eta .
$$

Substituting in (4.6), for any $u \in \mathcal{U}$ one has

$$
\vartheta(\bar{x}, \bar{y}) \leq \mathbb{E}\left[e^{-\lambda \theta^{u}} \vartheta\left(X_{\bar{x}}^{u}\left(\theta^{u}\right), e^{\lambda \theta^{u}} Y_{\bar{x}, \bar{y}}^{u}\left(\theta^{u}\right)\right)\right]-\eta e^{-\lambda T} .
$$

Since $\eta$ and $T$ do not depend on $u$, a minimisation over $u$ contradicts the DPP.

- Case 2: $g(\bar{x})=\bar{y}$. Assume by contradiction that

$$
-\partial_{y} \varphi(\bar{x}, \bar{y})<0 \quad \text { and } \quad \lambda \varphi+H\left(\bar{x}, \bar{y}, D_{x} \varphi, \partial_{y} \varphi, D_{x}^{2} \varphi\right)<0 .
$$

We can again define $r_{1}>0$ such that $(4.5)$ is satisfied in $B\left((\bar{x}, \bar{y}), r_{1}\right)$. Moreover there exists $\tilde{r}_{2}>0$ such that

$$
\varphi(\xi, \zeta) \leq \varphi\left(\xi, \zeta^{\prime}\right)
$$

for any $(\xi, \zeta),\left(\xi, \zeta^{\prime}\right) \in B\left((\bar{x}, \bar{y}), \tilde{r}_{2}\right)$ such that $\zeta \leq \zeta^{\prime}$. For any $u \in \mathcal{U}$ we define the stopping time $\theta^{u}$ as the first exit time of the process $\left(X_{\bar{x}}^{u}(s), e^{\lambda s} Y_{\bar{x}, \bar{y}}^{u}(s)\right)$ and $\left(X_{\bar{x}}^{u}(s), e^{\lambda s} \bar{y}\right)$ from the ball $B((\bar{x}, \bar{y}), \tilde{R})$ for $\tilde{R}:=\min \left(r, r_{1}, \tilde{r}_{2}\right)>0$. As for Case 1 , we can still say that a.s.

$$
\vartheta\left(X_{\bar{x}}^{u}\left(\theta^{u}\right), Y_{\bar{x}, \bar{y}}^{u}\left(\theta^{u}\right)\right) \geq \varphi\left(X_{\bar{x}}^{u}\left(\theta^{u}\right), Y_{\bar{x}, \bar{y}}^{u}\left(\theta^{u}\right)\right)+\eta^{\prime} .
$$

for some $\eta^{\prime}>0$ not depending on $u$. Therefore, observing that

$$
e^{\lambda s} Y_{\bar{x}, \bar{y}}^{u}(s) \geq e^{\lambda s} \bar{y}
$$

for any $s \geq 0$ and using Ito's formula, we get

$$
\begin{aligned}
\vartheta(\bar{x}, \bar{y})=\varphi(\bar{x}, \bar{y}) & \leq \mathbb{E}\left[\varphi\left(X_{\bar{x}}^{u}\left(\theta^{u}\right), e^{\lambda \theta^{u}} \bar{y}\right)\right] \leq \mathbb{E}\left[\varphi\left(X_{\bar{x}}^{u}\left(\theta^{u}\right), e^{\lambda \theta^{u}} Y_{\bar{x}, \bar{y}}^{u}\left(\theta^{u}\right)\right)\right] \\
& \leq \mathbb{E}\left[\vartheta\left(X_{\bar{x}}^{u}\left(\theta^{u}\right), e^{\lambda \theta^{u}} Y_{\bar{x}, \bar{y}}^{u}\left(\theta^{u}\right)\right]-\eta^{\prime},\right.
\end{aligned}
$$

which yields again to a contradiction of the DPP.

Remark 4.3. Unlike the case of finite time horizon (see $[4,11])$, the HJB equation (4.1) obtained by the DPP does not admit a unique solution. Indeed, one can observe that the function identically equal to zero is always a solution of (4.1). To overcome this problem we will restrict our domain to

$$
D:=\left\{(x, y) \in \mathbb{R}^{d+1}: y>g(x), y \in\left(-M_{g}, M_{g}\right)\right\} .
$$

and add a Dirichlet boundary condition for $y=M_{g}$. 
Let

$$
\Gamma_{1}:=\left\{(x, y) \in \bar{D}: y=M_{g}\right\} ; \quad \Gamma_{2}:=\{(x, y) \in \bar{D}: y=g(x)\} .
$$

Then the value function $\vartheta$ is a viscosity solution of the following problem

$$
\begin{aligned}
\lambda \vartheta+H\left(x, y, D_{x} \vartheta, \partial_{y} \vartheta, D_{x}^{2} \vartheta\right) & =0 & & \text { in } D, \\
-\partial_{y} \vartheta & =0 & & \text { on } \Gamma_{2}
\end{aligned}
$$

and satisfies additional

$$
\vartheta=M_{g} \text { on } \Gamma_{1}
$$

in a strong sense. We point out that in our case the strong constant Dirichlet boundary condition on $\Gamma_{1}$ is compatible with the homogeneus derivative condition on $\Gamma_{2}$. This prevents possible problems related with mixed boundary conditions at the junctions where different components of the boundary cross.

Now, we can establish the following comparison result:

Theorem 4.4. Let assumptions (H1)-(H2) be satisfied and let $\underline{\vartheta} \in U S C(\bar{D})$, $\bar{\vartheta} \in L S C(\bar{D})$ respectively a bounded sub-and super-solution to equation (4.8) in $\bar{D}$ such that

$$
\underline{\vartheta} \leq M_{g} \quad \text { and } \quad \bar{\vartheta} \geq M_{g} \quad \text { on } \Gamma_{1} .
$$

Then $\underline{\vartheta} \leq \bar{\vartheta}$ in $\bar{D}$.

The proof can be obtained by a modification of the arguments in $[22$, Theorem 2.1] which shows how to deal with the derivative conditions; see also [24]. We report here the main steps.

Proof. We set

$\tilde{b}(x, y, u):=\left(\begin{array}{c}b(x, u) \\ \lambda y\end{array}\right) \in \mathbb{R}^{d+1}$ and $\tilde{\sigma}(x, y, u):=\left(\begin{array}{c}\sigma(x, u) \\ 0 \ldots 0\end{array}\right) \in \mathbb{R}^{(d+1) \times p}$.

Further, let $x$ denote the variable in the augmented state space $\mathbb{R}^{M}$ with $M:=d+1$, i.e. $x \equiv(x, y) \in \mathbb{R}^{M}$ and $x_{M}=y$. Then the Hamiltonian $H$ in (4.2) is given by

$$
H(x, p, P):=\sup _{u \in U}\left(-\tilde{b}(x, u) \cdot p-\frac{1}{2} \operatorname{Tr}\left[\tilde{\sigma} \tilde{\sigma}^{T}(x, u) P\right]\right) .
$$

Thanks to the Lipschitz continuity of the function $g$ that defines the boundary $\Gamma_{2}$, we can observe that there exists $\mu>0$ such that for any $z \in \Gamma_{2}$ we have

$$
\bigcup_{0 \leq \xi \leq \mu} B(z-\xi, \xi \mu) \subset D^{C}
$$

where $D^{C}$ denotes the complementary of the set $D$; this corresponds to [22, condition (2.9)]. Let us define for positive $\alpha$ and $\beta$ the functions

$$
\underline{\vartheta}_{\alpha, \beta}(x):=\underline{\vartheta}(x)-\alpha\left(M_{g}-x_{M}\right)-\beta, \quad \bar{\vartheta}_{\alpha, \beta}(x):=\bar{\vartheta}(x)+\alpha\left(M_{g}-x_{M}\right)+\beta \text {. }
$$


Thanks to the non-negativity of $\left(M_{g}-x_{M}\right)$ for $y \in \bar{D}$ we have

$$
\underline{\vartheta}_{\alpha, \beta} \leq \underline{\vartheta} \quad \text { and } \quad \bar{\vartheta}_{\alpha, \beta} \geq \bar{\vartheta} \quad \text { on } \quad \bar{D}
$$

and in particular

$$
\underline{\vartheta}_{\alpha, \beta} \leq M_{g} \quad \text { and } \quad \bar{\vartheta}_{\alpha, \beta} \geq M_{g} \quad \text { on } \quad \Gamma_{1} .
$$

With an abuse of notation, we denote by $D \underline{\vartheta}, D \bar{\vartheta}, D^{2} \underline{\vartheta}, D^{2} \bar{\vartheta}$ the corresponding elements of the semijets of $\underline{\vartheta}$ and $\bar{\vartheta}$, see $[17$, Section 2$]$. We have

$$
\lambda \underline{\vartheta}_{\alpha, \beta}+H\left(x, D \underline{\vartheta}_{\alpha, \beta}, D^{2} \underline{\vartheta}_{\alpha, \beta}\right)-\lambda \underline{\vartheta}-H\left(x, D \underline{\vartheta}, D^{2} \underline{\vartheta}\right) \leq-\lambda \beta+\lambda \alpha M_{g}
$$

and

$$
\lambda \bar{\vartheta}_{\alpha, \beta}+H\left(x, D \bar{\vartheta}_{\alpha, \beta}, D^{2} \bar{\vartheta}_{\alpha, \beta}\right)-\lambda \bar{\vartheta}-H\left(x, D \bar{\vartheta}, D^{2} \bar{\vartheta}\right) \geq \lambda \beta-\lambda \alpha M_{g} .
$$

Moreover, we have

$$
-\partial_{x_{M} \underline{\vartheta}} \alpha, \beta=-\partial_{x_{M} \underline{\vartheta}}-\alpha, \quad-\partial_{x_{M}} \bar{\vartheta}_{\alpha, \beta}=-\partial_{x_{M}} \bar{\vartheta}+\alpha
$$

Therefore for any $\beta>0$ and $\alpha=\alpha(\beta)>0$ small enough, $\underline{\vartheta}_{\alpha, \beta}$ (resp. $\bar{\vartheta}_{\alpha, \beta}$ ) is a sub-solution (resp. super-solution) to (4.8) with the following modified boundary condition on $\Gamma_{2}$ :

$$
\left.-\partial_{x_{M}} \vartheta+\alpha \leq 0 \quad \text { (resp. }-\partial_{x_{M}} \vartheta-\alpha \geq 0\right) .
$$

In the sequel we prove a comparison principle between the sub-solution $\underline{\vartheta} \alpha, \beta$ and super-solution $\bar{\vartheta}_{\alpha, \beta}$ of the modified problem. The comparison result between $\underline{\vartheta}$ and $\bar{\vartheta}$ follows from taking the limit of $\alpha, \beta \rightarrow 0$. In order to simplify the notation we denote the sub- and super-solution of the modified problem also by $\underline{\vartheta}$ and $\bar{\vartheta}$.

Let

$$
\Phi_{\gamma}(x):=\underline{\vartheta}(x)-\bar{\vartheta}(x)-2 \gamma \log \left(1+\left|\left(x_{1} \ldots x_{M-1}\right)\right|^{2}\right) .
$$

In order to simplify the notation, from now on we will denote $|x|_{M-1}=$ $\left|\left(x_{1} \ldots x_{M-1}\right)\right|$. Thanks to the boundedness and the upper semicontinuity of $\underline{\vartheta}-\bar{\vartheta}$, the function $\Phi_{\gamma}$ admits a maximum point $\hat{x}_{\gamma}=\hat{x}$ in $\bar{D}$.

Let us assume that there exists a sequence $\left\{\gamma_{k}\right\}_{k \geq 0}$ such that $\gamma_{k} \rightarrow 0$ and the points $\hat{x}_{\gamma_{k}}$ approach the boundary $\Gamma_{1}$. In this case for any $x \in \bar{D}$ we have

$$
\begin{aligned}
\underline{\vartheta}(x)-\bar{\vartheta}(x) & =\lim \sup _{k \rightarrow \infty} \underline{\vartheta}(x)-\bar{\vartheta}(x)-2 \gamma_{k} \log \left(1+|x|_{M-1}^{2}\right) \\
& \leq \lim \sup _{k \rightarrow \infty} \underline{\vartheta}(\hat{x})-\bar{\vartheta}(\hat{x}) \leq 0 .
\end{aligned}
$$

the last inequality follows by the fact that any convergent subsequence of points $\left\{\hat{x}_{\gamma_{k}}\right\}$ converges to a point of $\Gamma_{1}$ where one has $\underline{\vartheta}-\bar{\vartheta} \leq 0$ by assumption. We obtain the same result if $\Phi_{\gamma_{k}}(\hat{x}) \leq 0$ for all $k$.

Therefore, in the sequel, we assume that there exists a $\bar{\gamma}$ small enough such that $d\left(\hat{x}, \Gamma_{1}\right)=\rho>0$ and $\underline{\vartheta}(\hat{x})-\bar{\vartheta}(\hat{x}) \geq \Phi_{\gamma}(\hat{x})>\rho$ for any $\gamma \leq \bar{\gamma}$ and some $\rho>0$ (with $d\left(\cdot, \Gamma_{1}\right)$ we have denoted the Euclidean distance function to $\Gamma_{1}$ ). Moreover, by classical doubling variable techniques it is standard to obtain a contradiction if $\hat{x}$ belongs to the interior of $\bar{D}$ (see for instance [27, Theorem 
7.3]). Such techniques have to be modified if $\hat{x}$ belongs to $\Gamma_{2}$. Thanks to the property (4.9) of the domain, the existence of a family of $C^{2}$ test functions $\left\{w_{\varepsilon}\right\}_{\varepsilon>0}$ can be proven as in [22, Theorem 4.1]. Among the other properties, $\left\{w_{\varepsilon}\right\}: \mathbb{R}^{M} \times \mathbb{R}^{M} \rightarrow \mathbb{R}$ satisfies:

$$
\begin{aligned}
& w_{\varepsilon}(x, x) \leq \varepsilon \\
& w_{\varepsilon}(x, y) \geq C \frac{|x-y|^{2}}{\varepsilon} \\
& -\partial_{x_{M}} w_{\varepsilon}(x, y) \geq-C \frac{|x-y|^{2}}{\varepsilon} \quad \text { if } x \in \Gamma_{2} \cap B(\hat{x}, \eta), y \in B(\hat{x}, \eta) \\
& -\partial_{y_{M}} w_{\varepsilon}(x, y) \geq 0 \quad \text { if } y \in \Gamma_{2} \cap B(\hat{x}, \eta), x \in B(\hat{x}, \eta)
\end{aligned}
$$

for $\varepsilon>0$ and some $\eta>0$ small enough.

Applying the doubling variables procedure we define

$\Phi_{\varepsilon}(x, y):=\underline{\vartheta}(x)-\bar{\vartheta}(y)-\gamma \log \left(1+|x|_{M-1}^{2}\right)-\gamma \log \left(1+|y|_{M-1}^{2}\right)-w_{\varepsilon}(x, y)-|x-\hat{x}|^{4}$.

and we denote by $\left(x_{\varepsilon}, y_{\varepsilon}\right)$ its maximum point. Thanks to properties (4.12) and (4.13), we have for $\varepsilon \rightarrow 0$ that

$$
x_{\varepsilon}, y_{\varepsilon} \rightarrow \hat{x} \quad \text { and } \quad \frac{\left|x_{\varepsilon}-y_{\varepsilon}\right|^{2}}{\varepsilon} \rightarrow 0
$$

Thus for $\varepsilon$ small enough we can assume that $x_{\varepsilon}, y_{\varepsilon} \notin \Gamma_{1}$, however since the case $\hat{x} \in \Gamma_{2}$ is taken into account, the sequence $\left(x_{\varepsilon}, y_{\varepsilon}\right)$ may consider points on $\Gamma_{2}$. Taking $\varepsilon$ small enough we can also say that $x_{\varepsilon}, y_{\varepsilon} \in B(\hat{x}, \eta)$ and then we can make use of properties (4.14) and (4.15).

If $x_{\varepsilon} \in \Gamma_{2}$, using (4.16), we have that for $\varepsilon$ small enough

$$
\begin{aligned}
& -\partial_{x_{M}}\left(w_{\varepsilon}\left(x_{\varepsilon}, y_{\varepsilon}\right)+\gamma \log \left(1+\left|x_{\varepsilon}\right|_{M-1}^{2}\right)+\left|x_{\varepsilon}-\hat{x}\right|^{4}\right) \\
& \geq-C \frac{\left|x_{\varepsilon}-y_{\varepsilon}\right|^{2}}{\varepsilon}-4\left|x_{\varepsilon}-\hat{x}\right|^{3}>-\alpha .
\end{aligned}
$$

Similarly if $y_{\varepsilon} \in \Gamma_{2}$ one has

$$
-\partial_{y_{M}}\left(-w_{\varepsilon}\left(x_{\varepsilon}, y_{\varepsilon}\right)-\gamma \log \left(1+\left|y_{\varepsilon}\right|_{M-1}^{2}\right)\right) \leq 0<\alpha
$$

for $\varepsilon$ small enough.

This means that, considering $w_{\varepsilon}\left(\cdot, y_{\varepsilon}\right)+\gamma \log \left(1+|\cdot|_{M-1}^{2}\right)+|\cdot-\hat{x}|^{4}$ and $-w_{\varepsilon}\left(x_{\varepsilon}, \cdot\right)-\gamma \log \left(1+|\cdot|_{M-1}^{2}\right)$ as test functions for $\underline{\vartheta}$ and $\bar{\vartheta}$ respectively, for sufficiently small values of $\varepsilon$ the derivative boundary conditions in $x_{\varepsilon}$ and $y_{\varepsilon}$ can be neglected and one can only consider in $\Gamma_{2} \cup D$

$$
\lambda \underline{\vartheta}+H\left(x_{\varepsilon}, D \underline{\vartheta}, D^{2} \underline{\vartheta}\right) \leq 0 \quad \text { and } \quad \lambda \bar{\vartheta}+H\left(y_{\varepsilon}, D \bar{\vartheta}, D^{2} \bar{\vartheta}\right) \geq 0
$$

in the viscosity sense. Thanks to the properties of $H$ and $w_{\varepsilon}$, this leads to a contradiction using the arguments in [22, 27].

Corollary 4.5. The value function $\vartheta$ is the unique bounded continuous viscosity solution to equation (4.8) in $\bar{D}$. 
Starting by its HJB characterization in $\bar{D}, \vartheta$ can be extended in a unique way to the full space $\mathbb{R}^{d} \times \mathbb{R}$ by

$$
\begin{aligned}
& \vartheta(x, y)=y \quad \text { for any } y \geq M_{g} \\
& \vartheta(x, y)=\vartheta(x, g(x)) \quad \text { for any } y \leq g(x) .
\end{aligned}
$$

However, we can observe that, in order to characterize the original value function $v$, the way we extend $\vartheta$ for $y>M_{g}$ does not matter and only the values of $\vartheta$ for $y=g(x) \leq M_{g}$ are relevant.

\section{Numerical APPROXimation}

In this section, convergence is proven for a general class of numerical schemes. Then, we show that a fully-discrete semi-Lagrangian method applied to the problem under consideration belongs to this class.

Let $B C$ denote the space of bounded and continuous functions in $\mathbb{R}^{d+1}$ equipped with the $L^{\infty}$-norm. Further, we introduce the discretization parameters

$$
\Delta x=\left(\Delta x_{1}, \ldots, \Delta x_{d}\right) \in\left(\mathbb{R}^{>0}\right)^{d}, \quad \Delta y>0, \quad \rho=(\Delta x, \Delta y)
$$

and denote the corresponding mesh by

$$
\mathcal{G}^{\rho}:=\left\{\left(x_{i}, y_{j}\right)=(i \Delta x, j \Delta y),(i, j) \in \mathbb{Z}^{d} \times \mathbb{Z}\right\},
$$

where $i \Delta x=\left(i_{1} \Delta x_{1}, \ldots, i_{N} \Delta x_{N}\right), i \in \mathbb{Z}^{d}$. We set

$$
\begin{aligned}
j_{x} & :=\min \{j \in \mathbb{Z} \mid j \Delta y \geq g(x)\}, \\
j_{M} & :=\min \left\{j \in \mathbb{Z} \mid j \Delta y \geq M_{g}\right\}
\end{aligned}
$$

and introduce a projection operator

$$
\Pi^{\mathcal{G}^{\rho}}(\phi)\left(x_{i}, y_{j}\right):= \begin{cases}y_{j} & \text { if } j \geq j_{M} \\ \phi\left(x_{i}, y_{j}\right) & \text { if } j_{M}>j \geq j_{x_{i}} \\ \phi\left(x_{i}, y_{j_{x_{i}}}\right) & \text { if } j<j_{x_{i}}\end{cases}
$$

for functions $\phi \in B C$. We aim to define a general approximation of the value function $\vartheta$. We start considering a general scheme

$$
S^{\rho}\left(x_{i}, y_{j}, \phi_{i, j}, \phi\right)=0
$$

that approximates the equation

$$
\lambda \phi+H\left(x, y, D_{x} \phi, \partial_{y} \phi, D_{x}^{2} \phi\right)=0
$$

at node $\left(x_{i}, y_{j}\right)$, with $H$ defined by (4.2). Here $\phi_{i, j}=\phi\left(x_{i}, y_{j}\right)$ and $\phi$ denote the values of $\phi$ at nodes different from $\left(x_{i}, y_{j}\right)$. $S^{\rho}$ may represent a finite difference operator (see $[13,12,30]$ ), or a semi-Lagrangian (SL) scheme $([32,15,19])$. The main idea of the numerical method described here is to mix the use of a standard scheme for (4.8a), together with a projection step on $\partial D$ in order to get the desired boundary conditions. Let us point out that a similar method was introduced for treating oblique derivative boundary conditions, i.e. the condition we have in $\Gamma_{2}$, in [5] for the case $g(x) \equiv|x|$ and in [11] for a general Lipschitz continuous function $g$. In addition, here 
the numerical solution has also to satisfy the Dirichlet condition on $\Gamma_{1}$. We define $V$ on $\mathcal{G}^{\rho}$ such that

$$
\left\{\begin{array}{lr}
V_{i, j}=y_{j} & \text { if } y_{j} \geq M_{g}, \\
S^{\rho}\left(x_{i}, y_{j}, V_{i j}, \Pi^{\mathcal{G}^{\rho}}(V)\right)=0 & \text { if } g\left(x_{i}\right) \leq y_{j}<M_{g}, \\
V_{i, j}=V_{i, j_{x_{i}}} & \text { if } y_{j}<g\left(x_{i}\right)
\end{array}\right.
$$

and we denote by $V^{\rho}$ its continuous extension by bilinear interpolation. We assume the grid $\mathcal{G}^{\rho}$ aligned with the domain $\left[-M_{g}, M_{g}\right]$, so that we have $V^{\rho}=M_{g}$ on $\Gamma_{1}$.

5.1. General convergence result. In order to prove the convergence of $V^{\rho}$ to the unique viscosity solution $\vartheta$ of (4.8) we will make use of the arguments introduced by Barles and Souganidis in [6]. These make use of the properties of stability, consistency and monotonicity of the scheme. We assume that the scheme defined by (5.4) satisfies the following assumptions:

(H3.a) Stability: for any $\rho$ the scheme (5.4) admits a solution $V^{\rho} \in B C(\bar{D})$. Moreover, there exist $M, L \geq 0$ such that

$$
V^{\rho}(x, y) \leq M \quad \text { and } \quad\left|V^{\rho}(x, y)-V^{\rho}\left(x, y^{\prime}\right)\right| \leq L\left|y-y^{\prime}\right|
$$

for any $(x, y),\left(x, y^{\prime}\right) \in \bar{D}$ and $\rho>0$.

(H3.b) Consistency: the scheme $S^{\rho}$ is consistent with respect to (4.1) in $\bar{D}$, i.e. for all $(x, y) \in \bar{D}$ and every $\phi \in C^{2}(\bar{D})$ there holds

$$
\operatorname { l i m } _ { \substack{ \substack {\rho \rightarrow 0, \bar{D} \ni(\xi, \gamma) \rightarrow{ \rho \rightarrow 0 , \\
\overline { D } \ni ( \xi , \gamma ) \rightarrow (\begin{subarray}{c}{\zeta \rightarrow 0 \\
\zeta \rightarrow y}, }}\end{subarray}} S^{\rho}(\xi, \gamma, \phi(\xi, \gamma)+\zeta, \phi+\zeta)=\lambda \phi+H\left(x, D_{x} \phi, D_{x}^{2} \phi\right) .
$$

(H3.c) Monotonicity: for every $\rho, r \in \mathbb{R},(x, y) \in \bar{D}, S^{\rho}(x, y, r, \phi)$ depends only on the values of $\phi$ in a neighborhood $B_{\eta(\rho)}(x, y)$ of $(x, y)$ with $\eta(\rho) \geq 0$ such that $\eta(\rho) \rightarrow 0$ for $\rho \rightarrow 0$. For all function $\phi_{1}, \phi_{2}: \mathbb{R}^{d} \times$ $\mathbb{R} \rightarrow \mathbb{R}$ with $\phi_{1} \geq \phi_{2}$ on $B_{\rho}(x, y)$, there holds

$$
S^{\rho}\left(x, y, r, \phi_{1}\right) \leq S^{\rho}\left(t, x, y, r, \phi_{2}\right) .
$$

Remark 5.1. Stability, consistency and monotonicity are the classical requirements to prove convergence of numerical schemes in the framework of viscosity solutions. However, in our case some slight modifications with respect to the original assumptions considerend in [6] are necessary. In particular, the additional regularity assumption with respect to the variable $y$ turns out to be fundamental to deal with Dirichlet boundary conditions, while the reason for our formulation of the monotonicity condition is the fact that the numerical scheme $S^{\rho}$ defined on $\bar{D}$ may use some values of the function $V^{\rho}$ outside the domain $\bar{D}$.

Theorem 5.2. Let assumptions (H1)-(H2) be satisfied and let the scheme (5.4) satisfy assumption (H3). Then for $\rho \rightarrow 0$ the solution $V^{\rho}$ converges to the unique viscosity solution to (4.1). 
Proof. Let us define for $(x, y) \in \bar{D}$

$$
\begin{aligned}
\bar{V}(x, y) & :=\limsup _{\substack{\bar{D} \ni(\xi, \gamma) \rightarrow(x, y) \\
\rho \rightarrow 0}} V^{\rho}(\xi, \gamma), \\
\underline{V}(x, y) & :=\liminf _{\substack{\bar{D} \ni(\xi, \gamma) \rightarrow(x, y) \\
\rho \rightarrow 0}} V^{\rho}(\xi, \gamma)
\end{aligned}
$$

Observe that the semi-limits are well-defined thanks to the boundedness of $V^{\rho}$ uniformly with respect to $\rho$. We start by proving that $\bar{V}$ is a viscosity sub-solution to equation (4.1).

Thanks to the Lipschitz continuity of $V^{\rho}$ with respect to $y$ (uniform with respect to $\rho$ ) and the fact that, by definition, $V^{\rho}(x, y)=M_{g}$ on $\Gamma_{1}$ one has for any $(\xi, \gamma) \in \bar{D}$

$$
\left|V^{\rho}(\xi, \gamma)-M_{g}\right|=\left|V^{\rho}(\xi, \gamma)-V^{\rho}\left(\xi, M_{g}\right)\right| \leq L\left|\gamma-M_{g}\right|
$$

so that

$$
\lim _{\substack{\bar{D} \ni, \gamma) \rightarrow(x, y) \\ \rho \rightarrow 0}} V^{\rho}(\xi, \gamma)=M_{g}
$$

Hence, $\bar{V}=M_{g}$ on $\Gamma_{1}$ and the Dirichlet condition is satisfied.

Let $\varphi \in C^{2}(\bar{D})$ and let $(\bar{x}, \bar{y})$ be a local maximum point for $\bar{V}-\varphi$ on $\bar{D}$. Without loss of generality we can assume that $(\bar{x}, \bar{y})$ is a strict local maximum in $B_{r}(\bar{x}, \bar{y}) \cap \bar{D}$ for a certain $r>0$ and $\varphi \geq 2 \sup _{\rho}\left\|W^{\rho}\right\|_{\infty}$ outside the ball $B_{r}(\bar{x}, \bar{y})$. We claim that

$$
\begin{cases}\lambda u+H\left(\bar{x}, \bar{y}, D_{x} \varphi, \partial_{y} \varphi, D_{x}^{2}\right) \varphi \leq 0 & \text { if }(\bar{x}, \bar{y}) \in D, \\ \min \left(\lambda u+H\left(\bar{x}, \bar{y}, D_{x} \varphi, \partial_{y} \varphi, D_{x}^{2} \varphi\right),-\partial_{y} \varphi\right) \leq 0 & \text { if }(\bar{x}, \bar{y}) \in \Gamma_{2} .\end{cases}
$$

We follow the argument in Barles and Souganidis [6]. There exists a sequence $\rho_{k},\left(x_{k}, y_{k}\right)$ such that $\rho_{k} \rightarrow 0,\left(x_{k}, y_{k}\right) \rightarrow(\bar{x}, \bar{y})$ for $k \rightarrow \infty$, and

$$
\left(V^{\rho_{k}}-\varphi\right)\left(x_{k}, y_{k}\right)=\max _{\bar{D}}\left(V^{\rho_{k}}-\varphi\right)=\delta_{k} \rightarrow 0, \quad \text { as } k \rightarrow \infty
$$

and

$$
V^{\rho_{k}}\left(x_{k}, y_{k}\right) \rightarrow \bar{V}(\bar{x}, \bar{y}), \quad \text { as } k \rightarrow \infty .
$$

Case 1: $(\bar{x}, \bar{y}) \in D$. For $k$ large enough $\left(x_{k}, y_{k}\right) \in D$. Since $g$ is continuous, for $\rho_{k}$ small enough we can assume that $y>g(x)$ for any $(x, y) \in B_{\eta\left(\rho_{k}\right)}\left(x_{k}, y_{k}\right)$ (where $B_{\eta\left(\rho_{k}\right)}\left(x_{k}, y_{k}\right)$ is the neighborhood that appears in assumption (H3.c)). Consequently, for $k$ big enough

$$
\Pi^{\mathcal{G}_{\rho_{k}}}\left(V^{\rho_{k}}\right)=V^{\rho_{k}} \quad \text { and } \quad V^{\rho_{k}}<\varphi+\delta_{k}
$$

in $B_{\eta\left(\rho_{k}\right)}\left(x_{k}, y_{k}\right)$. By the monotonicity of the scheme (assumption (H3.c)) we further deduce

$$
\begin{aligned}
0 & =S^{\rho_{k}}\left(x_{k}, y_{k}, V^{\rho_{k}}\left(x_{k}, y_{k}\right), \Pi^{\mathcal{G}_{\rho_{k}}}\left(V^{\rho_{k}}\right)\right) \\
& \left.\geq S^{\rho_{k}}\left(x_{k}, y_{k}, \varphi\left(x_{k}, y_{k}\right)+\delta_{k}, \varphi+\delta_{k}\right)\right)
\end{aligned}
$$


and by the consistency assumption (H3.b) we obtain that as $k \rightarrow \infty$

$$
\lambda \varphi+H\left(\bar{x}, \bar{y}, D_{x} \varphi, \partial_{y} \varphi, D_{x}^{2} \varphi\right) \leq 0 .
$$

- Case 2: $(\bar{x}, \bar{y}) \in \Gamma_{2}$. If $-\partial_{y} \varphi(\bar{x}, \bar{y}) \leq 0$ the sub-solution property on $\Gamma_{2}$ is automatically satisfied. Let us assume that $-\partial_{y} \varphi(\bar{x}, \bar{y})>0$.

We point out that if $\bar{y}=g(\bar{x}),\left(x_{k}, y_{k}\right)$ can also be on $\Gamma_{2}$ and the scheme may involve values $V_{m, n}$ on some point $\left(x_{m}, y_{n}\right)$ which is not in $\bar{D}$.

If $-\partial_{y} \varphi(\bar{x}, \bar{y})>0$, there exists a neighbourhood $\mathcal{V}$ of $(\bar{x}, \bar{y})$ where $\partial_{y} \varphi$ is well defined and $-\partial_{y} \varphi>0$. Therefore,

$$
y \leq y^{\prime} \Rightarrow \varphi(x, y) \geq \varphi\left(x, y^{\prime}\right) \quad \forall(x, y),\left(x, y^{\prime}\right) \in \mathcal{V} .
$$

and, taking $k$ large enough, $B_{\eta\left(\rho_{k}\right)}\left(x_{k}, y_{k}\right) \subset \mathcal{V}$. Let $(x, y) \in B_{\eta\left(\rho_{k}\right)}\left(x_{k}, y_{k}\right)$. If $y \geq g(x)$, we have

$$
\Pi^{\mathcal{G}_{\rho}}\left(V^{\rho_{k}}(x, y)\right)=V^{\rho_{k}}(x, y) \leq \varphi(x, y)+\delta_{k} .
$$

If $y<g(x), \Pi^{\mathcal{G}_{\rho}}\left(V^{\rho_{k}}(x, y)\right)=V^{\rho_{k}}\left(x, y_{j_{x}}\right)$ and we have

$$
\begin{aligned}
& \Pi^{\mathcal{G}_{\rho}}\left(V^{\rho_{k}}(x, y)\right)=V^{\rho_{k}}\left(x, y_{j_{x}}\right) \\
& \leq \varphi\left(x, y_{j_{x}}\right)+\delta_{k} \quad \text { using (5.6) } \\
& \leq \varphi(x, y)+\delta_{k} \quad \text { using (5.7) }
\end{aligned}
$$

For the last inequality one also need to observe that if $(x, y) \in \mathcal{V}$ and $y<g(x)$, thanks to the continuity of $g$ one can choose $k$ big enough so that also $\left(x, y_{j_{x}}\right) \in \mathcal{V}$.

Consequently, we have $\Pi^{\mathcal{G}_{\rho}}\left(V^{\rho_{k}}\right) \leq \varphi+\delta_{k}$ on $B_{\eta\left(\rho_{k}\right)}\left(x_{k}, y_{k}\right)$. Thus by monotonicity we have

$$
\begin{array}{r}
0=S^{\rho_{k}}\left(x_{k}, y_{k}, V^{\rho_{k}}\left(x_{k}, y_{k}\right), \Pi^{\mathcal{G}_{\rho}}\left(V^{\rho_{k}}\right)\right) \\
\left.\geq S^{\rho_{k}}\left(x_{k}, y_{k}, \varphi\left(x_{k}, y_{k}\right)+\delta_{k}, \varphi+\delta_{k}\right)\right)
\end{array}
$$

and using consistency when $k \rightarrow \infty$ we have

$$
\lambda \varphi+H\left(\bar{x}, \bar{y}, D_{x} \varphi, \partial_{y} \varphi, D_{x}^{2} \varphi\right) \leq 0 .
$$

This proves that $\bar{V}$ is a viscosity subsolution to (4.1). Analogously one can show that $\underline{V}$ is a viscosity sub-solution. Applying the comparison principle (Theorem 4.4), it follows that $\underline{V} \geq \bar{V}$ on $\bar{D}$. Since $\underline{V} \leq \bar{V}$ is always true by definition, it is possible to conclude that $\bar{V}=\underline{V}$ on $\bar{D}$, which proves the assertion.

5.2. Semi-Lagrangian scheme. In this section we introduce a semi-Lagrangian scheme and verify that it satisfies assumptions (H3). 
5.2.1. Semi-discretization. To simplify the presentation, we consider first a semi-discrete version of the scheme. Let $h>0$ be the time discretization parameter of the dynamics. We define the mapping $\mathscr{T}_{0}: B C \rightarrow B C$ with

$$
\begin{aligned}
& \mathscr{T}_{0}(\phi)(x, y):= \\
& \min _{u \in U}\left\{\frac{(1-\lambda h)}{2 p} \sum_{k=1}^{2 p} \phi\left(x+h b(x, u)+\sqrt{h p}(-1)^{k} \sigma_{\left\lfloor\frac{k+1}{2}\right\rfloor}(x, u), \frac{y}{1-\lambda h}\right)\right\}
\end{aligned}
$$

where $\lfloor\cdot\rfloor$ denotes the integer part. Furthermore, let $X\left(M_{g}\right)$ be the subset of $B C$ given by

$$
X\left(M_{g}\right):=\left\{\begin{array}{l|l}
\phi \in B C \cap \operatorname{Lip}_{y} \mid \begin{array}{l}
\phi(x, y)=y \text { for all }(x, y) \in \mathbb{R}^{d} \times \mathbb{R} \\
\text { with } M_{g} \leq y \leq 2 M_{g}(1-\lambda h)
\end{array}
\end{array}\right\}
$$

where $\operatorname{Lip}_{y}$ denotes the set of functions that are Lipschitz continuous with respect to $y$. We introduce the operator $T_{0}: X\left(M_{g}\right) \rightarrow X\left(M_{g}\right)$ defined by

$$
T_{0}(\phi)(x, y):=\mathscr{T}_{0}(\Pi(\phi))(x, y)
$$

where $\Pi$ is the the continuous version of the projection $\Pi^{\mathcal{G}^{\rho}}$, i.e. :

$$
\Pi(\phi)(x, y):= \begin{cases}y \wedge 2 M_{g} & \text { if } y \geq M_{g} \\ \phi(x, y) & \text { if } g(x) \leq y<M_{g} \\ \phi(x, g(x)) & \text { if } y \leq g(x)\end{cases}
$$

Remark 5.3. Compared with (5.3) here we have modified the value of the operator $\Pi$ for $y \geq M_{g}$ in order to guarantee the boundedness of the operator $T$. It is possible to verify that this does not affect the proof of the main convergence result which only concerns neighborhoords of points in $\bar{D}$.

Observe that for functions $\phi$ in $X\left(M_{g}\right)$ the operator $\Pi$ simplies to

$$
\Pi(\phi)(x, y)=\phi\left(x, g(x) \vee\left(y \wedge 2 M_{g}\right)\right) .
$$

Lemma 5.4. The operator $T_{0}$ is well-defined.

Proof. For every $\phi \in X\left(M_{g}\right)$ the image $T_{0}(\phi)$ is bounded and continuous. For $L \geq 0$ let $\operatorname{Lip}_{y}(L) \subset \operatorname{Lip}_{y}$ be the subset of functions with Lipschitz constant $L$. Then we have for $\phi \in \operatorname{Lip}_{y}(L)$

$$
\begin{aligned}
& \left|T_{0}(\phi)(x, y)-T_{0}(\phi)\left(x, y^{\prime}\right)\right| \\
& \leq \max _{u \in U} \frac{(1-\lambda h)}{2 p} \sum_{k=1}^{2 p}\left|\Pi(\phi)\left(X_{x}^{u, k, p}(h), Y_{y}^{u}(h)\right)-\Pi(\phi)\left(X_{x}^{u, k, p}(h), Y_{y^{\prime}}^{u}(h)\right)\right|
\end{aligned}
$$

where we use the notation

$$
\begin{aligned}
X_{x}^{u, k, p}(h) & :=x+h b(x, u)+\sqrt{h p}(-1)^{k} \sigma_{\left\lfloor\frac{k+1}{2}\right\rfloor}(x, u), \\
Y_{y}^{u}(h) & :=\frac{y}{1-\lambda h}
\end{aligned}
$$


for any $(x, y) \in \mathbb{R}^{d+1}$ and $u \in U$. From (5.11) we obtain

$$
\begin{aligned}
\left|\Pi(\phi)(x, y)-\Pi(\phi)\left(x, y^{\prime}\right)\right| & =\left|\phi\left(x, y \vee\left(g(x) \wedge 2 M_{g}\right)\right)-\phi\left(x, g(x) \vee\left(y^{\prime} \wedge 2 M_{g}\right)\right)\right| \\
& \leq L\left|\left(y \vee\left(g(x) \wedge 2 M_{g}\right)\right)-\left(y^{\prime} \vee\left(g(x) \wedge 2 M_{g}\right)\right)\right| \\
& \leq L\left|y-y^{\prime}\right|
\end{aligned}
$$

here we used the classical inequalitites

$$
\begin{aligned}
& |a \vee b-c \vee d| \leq|a-c| \vee|b-d|, \\
& |a \wedge b-c \wedge d| \leq|a-c| \vee|b-d| .
\end{aligned}
$$

Therefore, we have

$$
\begin{aligned}
\left|T_{0}(\phi)(x, y)-T_{0}(\phi)\left(x, y^{\prime}\right)\right| & \leq \max _{u \in U} \frac{(1-\lambda h)}{2 p} \sum_{k=1}^{2 p} L\left|Y_{y}^{u}(h)-Y_{y^{\prime}}^{u}(h)\right| \\
& \leq \max _{u \in U} \frac{(1-\lambda h)}{2 p} \sum_{k=1}^{2 p} L \frac{\left|y-y^{\prime}\right|}{(1-\lambda h)}
\end{aligned}
$$

which implies $T_{0}(\phi) \in \operatorname{Lip}_{y}(L)$.

Moreover, for $y \geq M_{g}$ one has $y /(1-\lambda h)>M_{g}$ and then

$$
T_{0}(\phi)(x, y)=\min _{u \in U}\left\{\frac{(1-\lambda h)}{2 p} \sum_{k=1}^{2 p}\left(\frac{y}{1-\lambda h}\right) \wedge 2 M_{g}\right\}=y \wedge 2 M_{g}(1-\lambda h)
$$

which proves that $T_{0}(\phi) \in X\left(M_{g}\right)$.

Next we verify the properties of stability, consistency and monotonicity (in the sense of assumption (H3)) for the scheme

$$
S^{h}(x, y, \phi(x, y), \phi):=\frac{1}{h}\left(\phi(x, y)-\mathscr{T}_{0}(\phi)(x, y)\right) \quad \text { for } \phi \in X\left(M_{g}\right) .
$$

The fact of dealing with an infinite horizon problem and therefore with a stationary PDE requires the use of a fixed point argument in order to prove the existence of a solution for (5.13). We point out that, due to the presence of the projection operator, $X\left(M_{g}\right)$ is the suitable space to guarantee the continuity of $T(\phi)$.

Lemma 5.5 (Stability). For $0 \leq \lambda h<1$ there exists a fixed point of the equation

$$
S^{h}(x, y, \phi(x, y), \Pi(\phi))=\frac{1}{h}\left(\phi(x, y)-T_{0}(\phi)(x, y)\right)
$$

in $X\left(M_{g}\right)$ and the scheme $S^{h}$ is stable in the sense of assumption (H3.a).

Proof. $T_{0}$ is a contraction on $X\left(M_{g}\right)$, since

$$
\left\|T_{0}(v)-T_{0}(w)\right\|_{\infty} \leq \frac{(1-\lambda h)}{2 p} \sum_{k=1}^{2 p}\|(\Pi v)-(\Pi w)\|_{\infty} \leq(1-\lambda h)\|v-w\|_{\infty} .
$$


Furthermore, since $\left(X\left(M_{g}\right),\|\cdot\|_{\infty}\right)$ is a closed subset of $\left(B C,\|\cdot\|_{\infty}\right)$, it is a complete metric space. Thus we obtain existence by the Banach-Cacioppoli fixed point theorem. Stability in the sense of assumption (H3.a) follows from the fact that the bound and Lipschitz constant of the fixed point do not depend on $h$.

The lemma implies in particular, that for any $(x, y) \in \bar{D}$ there exists a solution in $X\left(M_{g}\right)$ of the scheme

$$
S^{h}(x, y, \phi(x, y), \Pi(\phi))=0,
$$

which we denote by $V^{h}$ in the sequel. Observe that $V^{h}$ automatically satisfies the boundary condition $V^{h}\left(x, M_{g}\right)=M_{g}$.

Lemma 5.6 (Consistency). For any smooth and bounded function $\phi$, there exists some $C>0$ such that

$$
\left|\frac{1}{h}\left(\phi(x, y)-\mathscr{T}_{0}(\phi)(x, y)\right)-\left(\lambda \phi+H\left(x, y, D_{x} \phi, \partial_{y} \phi, D_{x}^{2} \phi\right)\right)\right| \leq C h .
$$

Proof. The assertion follows straightforward by a Taylor expansion. For simplicity we show the results in the one dimensional case. Observing that

$$
\frac{1}{1-\lambda h}=1+\lambda h+O\left(h^{2}\right)
$$

we have

$$
\begin{aligned}
& \phi(x, y)-\mathscr{T}_{0}(\phi)(x, y) \\
&= \phi(x, y)-\frac{(1-\lambda h)}{2} \min _{u \in U}\left\{\phi\left(x+h b(x, u)+\sqrt{h} \sigma(x, u), \frac{y}{1-\lambda h}\right)\right. \\
&\left.+\phi\left(x+h b(x, u)-\sqrt{h} \sigma(x, u), \frac{y}{1-\lambda h}\right)\right\} \\
&= \lambda h \phi(x, y)-\frac{(1-\lambda h)}{2} \min _{u \in U}\left\{\phi_{x}(x, y)(h b(x, u)+\sqrt{h} \sigma(x, u))+\phi_{y}(x, y)(\lambda h y)\right. \\
&+\frac{1}{2} \phi_{x x}(x, y)(h b(x, u)+\sqrt{h} \sigma(x, u))^{2}+\phi_{x y}(x, y)(h b(x, u)+\sqrt{h} \sigma(x, u))(\lambda h y) \\
&+\frac{1}{6} \phi_{x x x}(x, y)(h b(x, u)+\sqrt{h} \sigma(x, u))^{3} \\
&+\phi_{x}(x, y)(h b(x, u)-\sqrt{h} \sigma(x, u))+\phi_{y}(x, y)(\lambda h y) \\
&+\frac{1}{2} \phi_{x x}(x, y)(h b(x, u)-\sqrt{h} \sigma(x, u))^{2}+\phi_{x y}(x, y)(h b(x, u)-\sqrt{h} \sigma(x, u))(\lambda h y) \\
&\left.+\frac{1}{6} \phi_{x x x}(x, y)(h b(x, u)-\sqrt{h} \sigma(x, u))^{3}\right\}+O\left(h^{2}\right) \\
&= \lambda h \phi(x, y)-\min _{u \in U}\left\{h b(x, u) \phi_{x}(x, y)+h \lambda y \phi_{y}(x, y)+\frac{1}{2} h \sigma^{2}(x, u) \phi_{x x}(x, y)\right\}+O\left(h^{2}\right) .
\end{aligned}
$$

Lemma 5.7 (Monotonicity). For any $h>0$ with $0 \leq \lambda h<1$ the scheme is monotone in the sense of assumption (H3.c). 
Proof. For $(x, y) \in \bar{D}$ the definition of the operator $T$ involves only values at points $\left(x+h b(x, u)+\sqrt{h p}(-1)^{k} \sigma_{\left\lfloor\frac{k+1}{2}\right\rfloor}(x, u), y /(1-\lambda h)\right)$ which remain in a neighborhood $B_{\eta(h)}$ of $(x, y)$, with $\eta(h)=O(\sqrt{h})$. Moreover, by definition we have

$$
S^{h}(x, y, \phi(x, y), \phi)=\frac{1}{h}\left(\phi(x, y)-\mathscr{T}_{0}(\phi)(x, y)\right)
$$

and the assertion follows immediately, since for $\phi_{1} \leq \phi_{2}$ we have

$$
\mathscr{T}_{0}\left(\phi_{1}\right)(x, y) \leq \mathscr{T}_{0}\left(\phi_{2}\right)(x, y)
$$

Having verified the assumptions (H3), we can apply Theorem 5.2 and state a convergence result for the semi-discrete scheme.

Theorem 5.8. Let assumptions (H1)-(H2) be satisfied. Then for $h \rightarrow 0$ the solution $V^{h}$ of the scheme (5.14) converges uniformly to $\vartheta$ in $\bar{D}$.

5.2.2. Full-discretization. Now, we consider the scheme on the discretized space. This requires to introduce an interpolation step in order to define the value of the solution at points

$$
\left(x_{i}+h b\left(x_{i}, u\right)+\sqrt{h p}(-1)^{k} \sigma_{\left\lfloor\frac{k+1}{2}\right\rfloor}\left(x_{i}, u\right), \frac{y_{j}}{1-\lambda h}\right)
$$

that may not belong to the grid $\mathcal{G}^{\rho}(\rho \equiv(\Delta x, \Delta y))$. We denote by [ · ] the monotone, $P_{1}$ interpolation operator, satisfying the following properties:

(i) $[\phi]\left(x_{i}, y_{j}\right)=\phi\left(x_{i}, y_{j}\right), \quad \forall\left(x_{i}, y_{j}\right) \in \mathcal{G}^{\rho}$;

(ii) $|[\phi](x, y)-\phi(x, y)| \leq L|(\Delta x, \Delta y)|, \quad \forall \phi \in \operatorname{Lip}(L)$;

(iii) $|[\phi](x, y)-\phi(x, y)| \leq C\left(|\Delta x|^{2}+\Delta y^{2}\right)\left\|D^{2} \phi\right\|_{\infty} \quad \forall \phi \in C^{2}$;

(iv) $\phi_{1} \leq \phi_{2} \Rightarrow\left[\phi_{1}\right] \leq\left[\phi_{2}\right]$.

The fully discrete operator is then defined by

$$
\begin{aligned}
& \mathscr{T}(\phi)\left(x_{i}, y_{j}\right):= \\
& \min _{u \in U}\left\{\frac{(1-\lambda h)}{2 p} \sum_{k=1}^{2 p}[\phi]\left(x_{i}+h b\left(x_{i}, u\right)+\sqrt{h p}(-1)^{k} \sigma_{\left\lfloor\frac{k+1}{2}\right\rfloor}\left(x_{i}, u\right), \frac{y_{j}}{1-\lambda h}\right)\right\}
\end{aligned}
$$

and the fully discrete scheme reads:

$$
S^{\rho}\left(x_{i}, y_{j}, \phi\left(x_{i}, y_{j}\right), \Pi^{\mathcal{G}^{\rho}} \phi\right):=\frac{1}{h}\left(\phi\left(x_{i}, y_{j}\right)-\mathscr{T}\left(\Pi^{\mathcal{G}^{\rho}} \phi\right)\left(x_{i}, y_{j}\right)\right)
$$

where

$$
\Pi^{\mathcal{G}^{\rho}}(\phi)\left(x_{i}, y_{j}\right):= \begin{cases}y_{j} \wedge 2 M_{g} & \text { if } y_{j} \geq M_{g}, \\ \phi\left(x_{i}, y_{j}\right) & \text { if } g\left(x_{i}\right) \leq y_{j}<M_{g}, \\ \phi\left(x_{i}, g\left(x_{i}\right)\right) & \text { if } y_{j} \leq g\left(x_{i}\right) .\end{cases}
$$

We associate to each grid function $S^{\rho}\left(\cdot, \cdot, \phi(\cdot, \cdot), \Pi^{\mathcal{G}^{\rho}} \phi\right)$ the continuous function obtained by bilinear interpolation in the grid points and denote it by the same expression. 
Theorem 5.9. Let $0 \leq \lambda h<1$ and let the following condition be satisfied

$$
\frac{|\Delta x|^{2}+\Delta y^{2}}{h} \rightarrow 0 \quad \text { as } \quad \rho, h \rightarrow 0 .
$$

Then scheme (5.16) is stable in the subspace of piecewise linear functions in $X\left(M_{g}\right)$, consistent and monotone in the sense of definition (H3).

Proof. Stability follows by similar arguments as in the semi-discrete case. Indeed, observing that the bilinear interpolation preserves the Lipschitz constant, the fix point theorem still holds if we consider piecewise bilinear functions in $X\left(M_{g}\right)$. Consistency can follow by $(5.15)(\mathrm{iii})$. Indeed, when interpolation is taken into account, in the estimates obtained proving Lemma 5.6 one gets the extra term

$$
\frac{|\Delta x|^{2}+\Delta y^{2}}{h}\left\|\phi_{x x}\right\|
$$

which goes to zero guaranteeing the consistency property. Monotonicity is ensured by the use of a linear interpolation.

Corollary 5.10. Let $0 \leq \lambda h<1$ and let the following condition be satisfied

$$
\frac{|\Delta x|^{2}+\Delta y^{2}}{h} \rightarrow 0 \quad \text { as } \quad \rho, h \rightarrow 0 .
$$

Then, as $\rho, h \rightarrow 0$ the solution $V^{h, \rho}$ of the fully discrete scheme (5.16) converges in $\bar{D}$ to the unique viscosity of equation (4.8).

Proof. The proof follows from Theorem 5.9 and Theorem 5.2.

\section{A numerical test}

In this section we present a numerical result where the controlled system $X_{x}^{u}(\cdot)$ in $\mathbb{R}^{2}$ satisfies the following stochastic differential equation:

$$
\left\{\begin{array}{l}
\mathrm{d} X(s)=\left(\begin{array}{c}
X_{2}(s) \\
u(s)
\end{array}\right) \mathrm{d} s+\sigma(X(s)) \mathrm{d} B_{s}, s \geq 0 \\
X(0)=x
\end{array}\right.
$$

where $B$. is a two-dimensional Brownian motion $(p=2), U=[-1,1] \subset \mathbb{R}$ and the function $\sigma: \mathbb{R}^{2} \rightarrow \mathbb{R}^{2 \times 2}$ is defined, for every $x \equiv\left(x_{1}, x_{2}\right)^{T} \in \mathbb{R}^{2}$, by:

$$
\sigma(x)=\frac{1}{2} \max \left(1-\sqrt{\left(x_{1}+0.5\right)^{2}+\left(x_{2}-0.5\right)^{2}}, 0\right) \times\left(\begin{array}{ll}
1 & 0 \\
0 & 1
\end{array}\right) .
$$

This setting satisfies assumption (H1). We consider the cost function $g$ : $\mathbb{R}^{2} \rightarrow \mathbb{R}$ with:

$$
g(x):=\frac{1}{2} d(x, \mathcal{K}) \quad \forall x \in \mathbb{R}^{2}
$$


where $d(x, \mathcal{K})$ stands for the Euclidean distance between $x \in \mathbb{R}^{2}$ and the set $\mathcal{K}:=[-1,1]^{2}$. The auxiliary value function that will be approximated is given by:

$$
\vartheta(x, y)=\inf _{u \in \mathcal{U}} \mathbb{E}\left[\max _{s \in[0,+\infty)} e^{-\lambda s} d\left(X_{x}^{u}(s), \mathcal{K}\right) \vee y\right],
$$

with $\lambda=2$.

In this test, the computational domain for $\left(x_{1}, x_{2}, y\right)$ is

$$
\Upsilon:=[-2,2] \times[-2,2] \times[-1,1] .
$$

Observe that, given the boundedness of $\Upsilon$, the function $g$ satisfies assumption (H2).

The numerical scheme implemented is the SL scheme introduced in Section 5.2. We denote by $N_{x_{1}}, N_{x_{2}}$ and $N_{y}$ the number of mesh steps for the space variables $x_{1}, x_{2}$ and $y$ on the domain $\Upsilon$, i.e. $\Delta x_{1}=4 / N_{x_{1}}, \Delta x_{2}=$ $4 / N_{x_{2}}$ and $\Delta y=2 / N_{y}$. According to Section 5.2, we also use the notation $h$ for the time step in the SL scheme.

The control values $u$ are in $[-1,1]$ and since the dynamics depends linearly on the control, we consider in the numerical simulations only five control values $\left(N_{u}=5\right) u \in\{-1 ;-0.5 ; 0 ; 0.5 ; 1\}$ (actually, we have also verified that three control values $u \in\{-1 ; 0 ; 1\}$ give analogous results).

Since the exact value function is not known, we solve first the HJB equation on a refined grid where $N_{x_{1}}=N_{x_{2}}=640$ and $N y=160$ and with $h=0.015$. We denote by $V^{\text {ref }}$ the approximated solution obtained on this grid that we will consider as a reference solution. To check the stability and numerical convergence of the SL scheme, we consider different grids $\mathcal{G}^{\rho}$ $\left(\rho \equiv\left(\Delta x_{1}, \Delta x_{2}, \Delta y\right)\right)$ and time steps $h$, and compute the numerical solutions $V^{h, \rho}$. In Table 1, we report the numerical error $\left\|V^{\text {ref }}-V^{h, \rho}\right\|$ for different grids and norms. In this table, the first two columns indicates the grid size (i.e. $N_{x_{1}} \times N_{x_{2}} \times N_{y}$ ) and the SL time step $h$, respectively. In the other three columns, we report the computed error in $L^{1}, L^{2}$ and $L^{\infty}$-norm and the associated order of convergence.

Table 1. Convergence table.

\begin{tabular}{|c|c|cc|cc|cc|}
\hline Grid & $h$ & $L^{1}$-error & order & $L^{2}$-error & order & $L^{\infty}$-error & order \\
\hline \hline $40^{2} \times 10$ & 0.24 & $1.89 \mathrm{E}-01$ & - & $2.37 \mathrm{E}-01$ & - & $1.14 \mathrm{E}+00$ & - \\
$80^{2} \times 20$ & 0.12 & $9.71 \mathrm{E}-02$ & 0.96 & $1.34 \mathrm{E}-01$ & 0.83 & $7.28 \mathrm{E}-01$ & 0.64 \\
$160^{2} \times 40$ & 0.06 & $4.17 \mathrm{E}-02$ & 1.22 & $6.35 \mathrm{E}-02$ & 1.07 & $4.04 \mathrm{E}-01$ & 0.85 \\
$320^{2} \times 80$ & 0.03 & $1.41 \mathrm{E}-02$ & 1.57 & $2.26 \mathrm{E}-02$ & 1.49 & $1.73 \mathrm{E}-01$ & 1.23 \\
\hline
\end{tabular}

From this table, one can notice that the SL scheme is stable in $L^{\infty}$-norm. This confirms the theoretical results of the previous sections (compare these results with Lemma 5.6 and Theorem 5.9 which give for $|\Delta x|, \Delta y \sim h$ order one of consistency). Moreover, we observe that the numerical convergence is also valid in $L^{1}$ and $L^{2}$ norms. The observed asymptotic rate of convergence, 
for this example, seems to tend towards an order greater than one (however, this observation might be due to the fact that we are comparing with a numerical reference solution and not with the exact solution itself).

Figure 1 shows the approximation of $\vartheta\left(x_{1}, x_{2}, 0\right)=v\left(x_{1}, x_{2}\right)$ (where $v$ is defined as in (2.4)) obtained on different grids. More precisely, figure 1-(a) corresponds to an approximation of $\vartheta(\cdot, 0)$ on a grid of $20 \times 20 \times 5$ points and with $h=0.48$. In figure 1 -(b) the approximation of $\vartheta(\cdot, 0)$ is computed on a grid of $40 \times 40 \times 10$ points and with $h=0.24$. And finally, we display in figure 1-(c) an approximation of $\vartheta(\cdot, 0)$ on a grid of $80 \times 80 \times 20$ points and with $h=0.12$. From these plots, we notice again that the numerical scheme is stable.

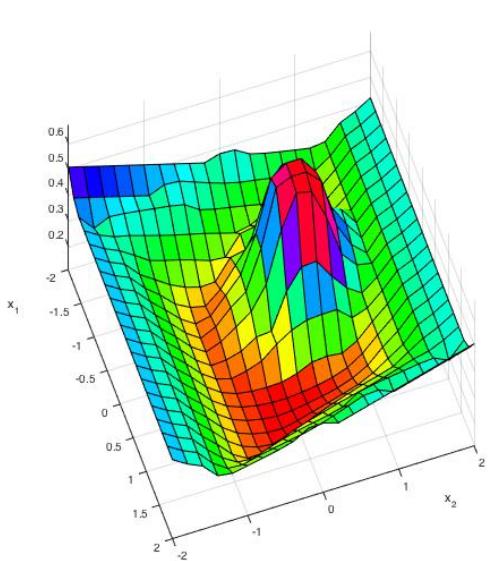

(a)

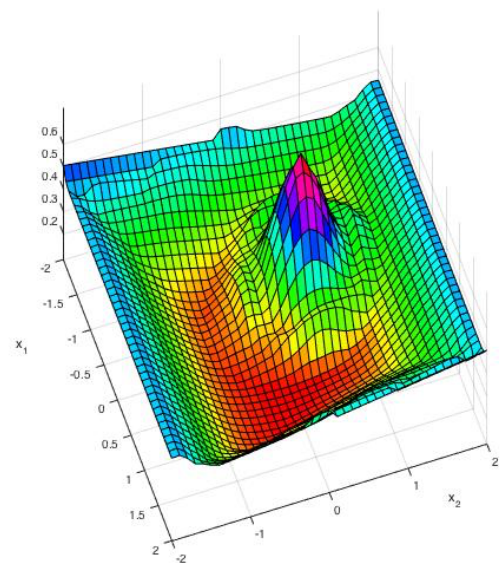

(b)

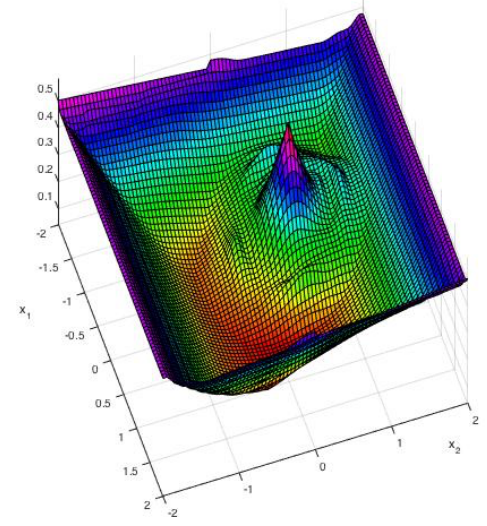

(c)

Figure 1. Approximation of $\vartheta(\cdot, \cdot, 0)$ on three different grids.

\section{Conclusions}

In this paper we have studied infinite horizon stochastic optimal control problems with cost in maximum form. By the introduction of an auxiliary 
Markovian problem and dynamic programming arguments we have characterized the associated value function by means of a HJB equation with mixed Dirichlet-derivative boundary conditions. We have proposed a general numerical scheme which incorporates the treatment of the boundary condition and proved its convergence to the unique viscosity solution of the HJB equation under the assumptions of monotonicity, consistency and stability. Furthermore, we have shown that a particular semi-Lagrangian scheme satisfies such assumptions and therefore can be used to approximate the value function of the original problem.

Further directions of work might involve the application of our scheme to the computation of viable and invariant sets (see [11, Section 2.3]) as well as the theoretical proof of the associated rate of convergence.

\section{REFERENCES}

1. O. Alvarez and E.N. Barron, Homogenization in $L^{\infty}$, J. of Differential Equations 183 (2002), 132-164.

2. L. S. Aragone and R. L. V. González, A Bellman's equation for minimizing the maximum cost, Indian J. of Pure and Applied Mathematics 31 (2000), no. 12, 1621-1632.

3. M. Assellaou, O. Bokanowski, A. Désilles, and H. Zidani, Value function and optimal trajectories for a maximum running cost control problem with state constraints. application to an abort landing problem., ESAIM: M2AN (2018), to appear.

4. G. Barles, C. Daher, and M. Romano, Optimal control on the $l^{\infty}$ norm of a diffusion process, SIAM J. Control Optim. 32 (1994), no. 3, 612-634.

5. Convergence of numerical schemes for parabolic equations arising in finance theory, M3AS 5 (1995), no. 1, 125-143.

6. G. Barles and P.E. Souganidis, Convergence of approximation schemes for fully nonlinear second order equations, Asymptotic Analysis 4 (1991), 271-283.

7. E. N. Barron, The Bellman equation for control of the running max of a diffusion and applications to lookback options, Applicable Analysis 48 (1993), 205-222.

8. _ Viscosity solutions and Analysis in $L^{\infty}$, Proceeding of the NATO advanced study Institute (1999), 1-60.

9. E. N. Barron and H Ishii, The Bellman equation for minimizing the maximum cost, Nonlinear Analysis 13 (1989), no. 9, 1067-1090.

10. P. Bettiol and F. Rampazzo, control problems as dynamic differential games, Nonlinear Differential Equations and Applications NoDEA 20 (2013), no. 3, 895-918.

11. O. Bokanowski, A. Picarelli, and H. Zidani, Dynamic programming and error estimates for stochastic control problems with maximum cost, Applied Mathematics and Optimization 71 (2015), no. 1, 125-163.

12. J.F. Bonnans, E. Ottenwaelter, and H. Zidani, Numerical schemes for the two dimensional second-order HJB equation, ESAIM: M2AN 38 (2004), 723-735.

13. J.F. Bonnans and H. Zidani, Consistency of generalized finite difference schemes for the stochastic HJB equation, SIAM J. Numerical Analysis 41 (2003), no. 3, 1008-1021.

14. B. Bouchard and N. Touzi, Weak dynamic programming principle for viscosity solutions, SIAM J. Control and Optimization 49 (2011), no. 3, 948-962.

15. F. Camilli and M. Falcone, An approximation scheme for the optimal control of diffusion processes, RAIRO Modél. Math. Anal. Numér. 29 (1995), no. 1, 97-122.

16. I. Capuzzo-Dolcetta, On a discrete approximation of the hamilton-jacobi equation of dynamic programming, Applied Mathematics and Optimization 10 (1983), 367-377. 
17. M.G. Crandall, H. Ishii, and P.L. Lions, User's guide to viscosity solutions of second order partial differential equations, Bulletin of the American Mathematical Society 27 (1992), no. 1, 1-67.

18. M.G. Crandall and P.L. Lions, Convergent difference schemes for nonlinear parabolic equations and mean curvature motion, Mathematics of Computation 75 (1996), no. 1, $17-41$.

19. K. Debrabant and E.R. Jakobsen, Semi-Lagrangian schemes for linear and fully nonlinear diffusion equations, Mathematics of Computation 82 (2012), no. 283, 14331462 .

20. S. C. Di Marco and R. L. V. González, A minimax optimal control problem with infinite horizon, Tech. report, Rapport de Recherche N. 2945, INRIA, Rocquencourt, 1996.

21. Supersolutions and subsolutions techniques in a minimax optimal control problem with infinite horizon, Indian J. of Pure and Applied Mathematics 29 (1998), no. 10, 1983-1998.

22. P. Dupuis and H. Ishii, On oblique derivative problems for fully nonlinear second-order elliptic PDE's on domains with corners, Hokkaido Mathematical Journal 20 (1991), no. $1,135-164$.

23. D. Goreac and O.-S. Serea, Linearization techniques for $L^{\infty}$-control problems and dynamic programming principles in classical and $L^{\infty}$-controlproblems, ESAIM:COCV 18 (2012), 836-855.

24. L. Grüne and A. Picarelli, Zubov's method for controlled diffusions with state constraints, Nonlinear Differential Equations and Applications 22 (2015), no. 6, 17651799.

25. L. Grüne and H. Zidani, Zubov's equation for state-constrained perturbed nonlinear systems, Math. Control Related Fields 5 (2015), no. 1, 55 - 71.

26. A. C. Heinricher and R. H. Stockbridge, Optimal control of the running max, SIAM J. Control and Optimization 29 (1991), no. 4, 936-953.

27. H. Ishii, On uniqueness and existence of viscosity solutions of fully nonlinear secondorder elliptic PDEs, Communications in Pure and Applied Mathematics 42 (1989), $14-45$.

28. __ Fully nonlinear oblique derivative problems for nonlinear Second-order elliptic PDEs, Duke Math. J. 62 (1991), no. 3, 633-661.

29. M. Jones and M. Peet, Solving dynamic programming with supremum terms in the objective and application to optimal battery scheduling for electricity consumers subject to demand charges, IEEE 56th Annual Conference on Decision and Control (CDC), 2017.

30. H.J. Kushner and P. Dupuis, Numerical methods for stochastic control problems in continuous time, Springer Verlag, New York.

31. P.L. Lions, Optimal control of diffusion processes and Hamilton-Jacobi-Bellman equations. Part I: The Dynamic Programming principle and applications, Communications in Partial Differential Equations 8 (1983), no. 10, 1101-1174.

32. J. L. Menaldi, Some estimates for finite difference approximations, SIAM J. Control and Optimization 27 (1989), 579-607.

33. R. Munos and H. Zidani, Consistency of a simple multidimensional scheme for Hamilton-Jacobi-Bellman equations, Comptes Rendus Mathématique 340(7) (2005), 499-502.

34. M. Quincampoix and O.-S. Serea, A viability approach for optimal control with infimum cost, Annals of the "Alexandru Ioan Cuza" University of Iasi 48 (2002), no. 1, 113-132.

35. O-S. Serea, Discontinuous differential games and control systems with supremum cost, Journal of Mathematical Analysis and Applications 270 (2002), no. 2, $519-542$.

36. N. Touzi, Optimal stochastic control, stochastic target problems, and backward SDE, Fields Institute Monographs, vol.49, Springer, 2012. 
37. J. Yong and X.Y. Zhou, Stochastic Controls: Hamiltonian Systems and HJB Equations, Applications of mathematics, Springer New York, 1999.

Department of Mathematics, Humboldt University of Berlin, Berlin, Germany; CMAP, Ecole Polytechnique, CNRS, Université Paris Saclay, and Inria, FRANCE

Email address: axel.kroener@inria.fr

Department of Mathematics, Imperial College, Huxley Building, SW72AZ, LONDON, UK

Email address: a.picarelli@imperial.ac.uk

ensta Paristech, 828, Boulevard des Maréchaux, 91762 Palaiseau Cedex, FRANCE

Email address: hasnaa.zidani@ensta-paristech.fr 